\title{
Absolute Configuration Determination by Quantum Mechanical Calculation of Chiroptical Spectra: Basics and Applications to Fungal Metabolites
}

\author{
Stefano Superchi*a, Patrizia Scafato ${ }^{\mathrm{a}}$, Marcin Górecki ${ }^{\mathrm{b}}$ and Gennaro Pescitelli*b \\ ${ }^{a}$ Dipartimento di Scienze, Università della Basilicata, Potenza, Italy; ${ }^{b}$ Dipartimento di Chimica e Chimica Industriale, \\ Università di Pisa, Pisa, Italy
}

\begin{abstract}
The application of quantum mechanical simulation of chiroptical properties, i.e. electronic circular dichroism (ECD), optical rotation (OR), and vibrational circular dichroism (VCD), to the assignment of the absolute configuration of chiral naturally occurring metabolites of fungal origin, is reviewed. The fundamentals of such chiroptical spectroscopies as well as the specific experimental and computational issues allied to the application of their ab initio calculation is reported. Some examples, related to the use of the ECD, VCD, and OR techniques and highlighting the practical application of the methods, are also described.
\end{abstract}

Keywords: structure elucidation; natural products; conformational analysis; electronic circular dichroism; vibrational circular dichroism; optical rotation; density functional theory

\section{INTRODUCTION}

Chirality is ubiquitous in living organisms. The three most important and most abundant natural products, peptides, nucleic acids and carbohydrates, are all chiral. As a consequence, chirality is of prime importance in the interaction of all living matters with their environment. In general, the different stereoisomers of any biologically active compound are expected to interact differently with the receptors, transport proteins, membranes etc. of living organisms. This is also true for the two optical antipodes or enantiomers of any chiral compound, i.e. two molecules related by mirror-image relationship which differ only in their absolute configuration. The two enantiomers of a chiral substance show in general different pharmacology, different pharmacokinetics as for all ADME parameters (absorption, distribution, metabolism, excretion), and different toxicology.[1] While the desired biological activity is often associated with only one enantiomer (called eutomer), the other enantiomer (called distomer) may be less effective, or totally ineffective, or have unwanted effects, or even be an antagonist of the eutomer. At least, the distomer will be an "isomeric ballast" for the organism and the environment.[2] The impact of chirality on pharmaceutics and medicine has been recognized since 1992 by worldwide pharmaceutical regulatory agencies, which nowadays require a full stereochemical characterization including the absolute configuration of chiral molecules to be considered as pharmaceuticals.[3, 4] Interestingly enough, an increasingly larger portion of chiral drugs is patented as single enantiomer (about 90\% between 2004-2006 in USA). [5] The number and importance of currently marketed chiral drugs is

*Address correspondence to S. S. at Dipartimento di Scienze,

Università della Basilicata, Viale dell'Ateneo Lucano, 10, 85100

Potenza, Italy; E-mail: stefano.superchi@unibas.it; to G. P. at

Dipartimento di Chimica e Chimica Industriale, Università di Pisa, Via

Moruzzi 13, 56124 Pisa, Italy; E-mail gennaro.pescitelli@unipi.it already remarkable,[6] and a recent report by Global Industry Analysts has foreseen that nearly $95 \%$ of all drugs will be chiral by 2020.[7]

Several important therapeutic agents are either natural products or semi-synthetically derived thereof.[8, 9] The number of new small-molecule drugs directly or indirectly derived from natural products has been roughly constant over the last 34 years and amounts to an average $33 \%$ of the total. In the area of anticancer drugs, in particular, the percentage grows to $49 \%$.[10] As far as plants are concerned as a source,[11] there is growing evidence that a large number of bioactive compounds previously believed to be plant-derived, are in fact produced either directly or through horizontal gene transfer by endophytic microbes, most often fungi.[12, 13] Above $80 \%$ of all known natural products are chiral, and most have multiple centers of chirality.[14] Although it is commonly believed that only a single stereoisomer (or enantiomer) is produced and biologically active, occasionally both enantiomers are formed[15] and sometimes enantiomeric mixtures are produced.[16]

The short discussion above intends to demonstrate that any time a new natural compound is discovered, especially when it is investigated for bioactivity and potential pharmacological activity, its structure elucidation must necessarily include a full stereochemical assignment, that is, the determination of the relative and absolute configuration ( $\mathrm{RC}$ and AC, respectively). Most often, however, this is the most difficult step of the whole structure elucidation. This statement may sound surprising in the case of $\mathrm{AC}$ assignment, because obviously "there are only two possible conclusions in absolute configurational studies: the conclusion is either correct or wrong".[17] However, such a piece of information is seldom available in a straightforward

(C) 2014 Bentham Science Publishers 
and unambiguous way for previously unknown compounds, as we will discuss in the next section. It is hard to believe that the first method for the assignment of $\mathrm{AC}$ without the necessity of an external reference (see section 2.3) was established only in 1951 with the X-ray diffraction experiment of Bijvoet et al. on NaRb (+)-tartrate.[18] For several decades, the problem of $\mathrm{AC}$ assignment has been based either on the Bijvoet method or on chemical correlations with known compounds.[19] Only relatively recently, assigning ACs has become the first purpose of chiroptical spectroscopies, that is, those phenomena arising from the interaction of circularly polarized light with chiral non-racemic matter.[20, 21] These include the three techniques which will be the main object of the present review, namely electronic circular dichroism (ECD), vibrational circular dichroism (VCD), and optical rotation (OR). In the last 15 years, chiroptical spectroscopies have experienced a "renaissance"[22] thanks to the development of computational power, which has made possible to calculate ECD/VCD/OR of real-life molecules such as natural products, with high-level quantum mechanical (QM) methods and in short times (Figure 1). The topic of the present review is to discuss the principles and examples of application of $\mathrm{ECD} / \mathrm{VCD} / \mathrm{OR}$ calculations of fungal metabolites as a tool to assign their AC. Several excellent reviews are already available on the $\mathrm{QM}$ simulations of $\mathrm{ECD} / \mathrm{VCD} / \mathrm{OR}$ with special reference to natural products, focusing either on a single technique or on a combination thereof.[23-34] We will necessary follow the same pattern of these reviews: it is in fact important to stress that, from the viewpoint of structural elucidation, a fungal metabolite does not behave differently from any other low-molecular-weight natural product. On the contrary, thanks to their rich structural diversity, we can take fungal metabolites as representative of the whole class of natural products with non-obvious AC (i.e. excluding proteins, nucleic acids and carbohydrates).
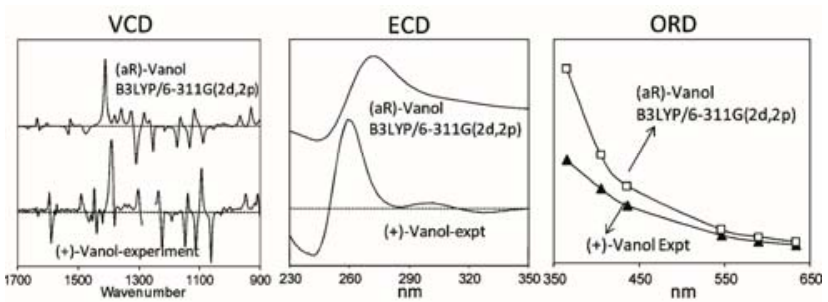

FIGURE 1: Calculated (top curves) and experimental (bottom curves) VCD, ECD, and ORD spectra of $(+)-(\mathrm{a} R)-$ 3,3'-diphenyl-[2,2'-binaphthalene]-1,1'-diol (VANOL). Reprinted with permission from [35]. Copyright 2009 American Chemical Society.

\section{STRUCTURE ELUCIDATION OF FUNGAL METABOLITES AND NATURAL PRODUCTS FROM OTHER SOURCES}

In this section we will briefly summarize the main techniques used to elucidate the structure of novel natural products, in particular fungal metabolites. We will focus especially on the determination of the stereochemistry, that is, the $\mathrm{RC}$ and $\mathrm{AC}$.

\subsection{Determine constitution}

The first step in the structural elucidation of a newly isolated natural product is the determination of the constitution or the "planar structure". This is normally achieved by a combination of spectroscopic techniques, with a major role played by nuclear magnetic resonance (NMR).[36] First, mass spectrometry (MS) provides the molecular formula from high resolution mass spectra (HRMS). The molecular formula immediately yields the degree of unsaturation, i.e. the number of unsaturations and/or rings, which helps a lot in the analysis of NMR spectra. The molecular formula is also essential to reveal "dimeric" symmetric structures which cannot be ascertained by NMR; to this purpose, however, proper MS ionization methods are necessary. IR and UV-Vis spectroscopies are then sought for the presence of signals characteristic of functional groups such as carbonyl, conjugated double bonds, and aromatic or hetero-aromatic systems.

Mono-dimensional ${ }^{1} \mathrm{H}$ and ${ }^{13} \mathrm{C}$ NMR spectra, and the use of DEPT sequence (distortion enhancement by polarization transfer) with variable pulse angles, are first scanned to identify the number and nature of carbons and protons. Various multi-dimensional NMR techniques are then used to recognize the diverse molecular fragments and their connections.[37] These techniques include ${ }^{1} \mathrm{H}-{ }^{1} \mathrm{H}$ COSY (correlation spectroscopy), TOCSY (totally correlated spectroscopy), ${ }^{1} \mathrm{H}_{-}{ }^{13} \mathrm{C}$ HMQC (heteronuclear multiple quantum correlation), and HMBC (heteronuclear multiple bond coherence). In the presence of vicinal tertiary carbon atoms, the connection between various fragments may not be obtained directly e.g. by COSY, TOCSY or HMBC, and must be deduced indirectly. Computer-aided structure elucidation (CASE) based on COSY/HMBC data is nowadays possible for relatively large molecules.[38] Finally, selective nuclear Overhauser effects (NOE), NOESY (NOE spectroscopy) and ROESY (rotating-frame Overhauser effect spectroscopy) spectra reveal the spatial proximity between protons and may be used to check the proposed structure, although their main application is in the study of molecular conformation and RC (vide infra).

Despite the versatility and broad applicability of spectroscopic tools, wrong structures of natural products are often encountered.[39, 40] Most errors occur because of an incorrect molecular formula, or when NMR signals are overlapped, or for molecules with a too low $\mathrm{H}$-to- $\mathrm{C}$ ratio to obtain proper ${ }^{1} \mathrm{H} /{ }^{13} \mathrm{C}$ correlations. A definite proof of the 
structure may be offered by X-ray crystallography and total synthesis. Both methods have known disadvantages, moreover X-ray crystallography can only be applied to suitable crystalline materials which appear to be the minority in the field of natural products.[41]

\subsection{Assign relative configuration}

Most natural products, including fungal metabolites, have multiple chiral centers, with an average of 5.2 chiral carbons per compound.[14] It is therefore mandatory to determine the relative stereochemistry of all stereogenic elements before proceeding with the absolute stereochemistry assignment. The determination of the RC is strictly related to the study of the molecular conformation. Both aspects depend in fact on experimental or computational data sensitive to the threedimensional structure. These latter include NMR data such as NOE effects, scalar J-couplings (especially ${ }^{3} J_{\mathrm{HH}}$ and ${ }^{3} J_{\mathrm{CH}}$ ), and ring-current shifts.[42] The coupling constants of vicinal and sometimes more distant nuclei is dictated by the mutual dihedral angles. A rough quantitative estimate is possible by means of Karplus-type equations, which however should be applied carefully because of their dependence on the nature and hybridization of attached atoms.[43] Moreover, it must be kept in mind that measured NOE effects and J-couplings are average values over the whole conformational ensemble. This is especially crucial for NOE, because of the sixthpower scaling with distance, so that a poorly populated conformation may be responsible for an apparent NOE. This complicates a lot the RC determination of flexible molecules or of flexible fragments (e.g. long alkyl chains). It is therefore recommended to complement the experimental data with the results of molecular modeling (vide infra).

Previously misassigned configuration of one or more chiral centers, in molecules with multiple chirality centers, is by far the most common reason for the structural revision of natural products.[39, 40, 44] Especially in this context, X-ray crystallography and total synthesis represent the ultimate tools for a correct structural elucidation.

\subsection{Assign absolute configuration}

As we have already stressed before, the structure elucidation of any chiral molecule may be said to be complete only when its absolute stereochemistry is determined. Very often, this is the most intriguing step of the whole process, as proven by the fact that a lot of published structures of novel natural products have undetermined $\mathrm{AC}$, not to mention the several AC revisions appeared in years. $[40,45]$ Two large families of techniques for assigning ACs may be recognized: relative methods and absolute methods.

\subsubsection{Relative methods:}

These methods require the existence of a reference compound, which may consist in: a) the same compound under investigation, if previously known including its AC; b) a compound with known $\mathrm{AC}$ which is structurally similar to the target compound, or can be chemically transformed into or prepared from the target compound; c) a molecular portion with known configuration which can be attached to the sample to provide a suitable derivative to be further characterized.

The most obvious way to assign ACs is to compare the measured value of optical rotation (OR) of the target compound with that of the pure or enantioenriched sample. OR should always be measured, at least at the sodium $\mathrm{D}$ line $(589.3 \mathrm{~nm})$, for any novel natural product. A non-zero $[\alpha]_{\mathrm{D}}$ value indicates a chiral non-racemic substance, although the opposite is not necessarily true. Of the around 282,000 entries comprised in the Dictionary of Natural Products (DNP) as of August 2016,[46] 41.5\% have a reported nonzero $\mathrm{OR}$ value. However, $\mathrm{OR}$ is very sensitive to the conditions of measurements (solvent, temperature, concentration) and it is compulsory to compare data obtained in similar conditions, otherwise erroneous assignments may occur.[44]

Other relative methods include so-called stereochemical correlations, that is, chemical transformations which occur by known stereochemical pathways between compounds with established stereochemistry. These include total syntheses of natural products based on the use of stereodefined chiral reactants. Most "classical" assignments of AC were based on such approach.[19]

The use of an "internal reference", i.e. a fragment or reactant of known AC, makes it possible for "chirally blind" techniques such as NMR and MS to discriminate between enantiomers. While MS is mostly used to estimate enantiomeric excesses,[47] NMR has also found application in the AC determination. This application is based on the use of so-called chiral derivatizing agents (CDAs) of known AC.[48] A well-known example is the derivatization of chiral alcohols with the Mosher reagent. The main drawback of these approaches is that no universal CDA exists and the most suitable one for a specific sample must be found on a case-by-case basis. Moreover, the spectrum-AC correlation is essentially semi-empirical and then leading to ambiguous assignments when dealing with molecules having particular conformational situations.

\subsubsection{Absolute methods:}

These methods are independent of any reference compound for assigning the $\mathrm{AC}$ of a novel compound. As such, they are intrinsically more versatile than relative methods and therefore of special interest in the context of natural products, because of the structural diversity of these latter.

The most important absolute technique for assigning ACs is the X-ray analysis of crystalline compounds exhibiting anomalous dispersion.[49] The method dates back to the already quoted Bijvoet's experiment on $\mathrm{NaRb}(+)$ tartrate.[18] The main prerequisites of the method are the 
availability of suitable single crystals and the presence of at least a strong scatterer, i.e. a "heavy" atom, such as Br, P or $\mathrm{S}$ for organic compounds (but $\mathrm{F}$ or even $\mathrm{O}$ may suffice with very careful work). Both prerequisites are far from trivial for natural products. For example, only $7 \%$ of the 282,000 entries in the DNP contain atoms other than $\mathrm{H} / \mathrm{C} / \mathrm{O} / \mathrm{N} / \mathrm{F}$.[46] Of the 813,000 entries in the Cambridge Structural Database (CSD) for X-ray structures (last updated in February 2016), [50] less than 3\% appear to be natural products other than proteins or nucleic acids. Moreover, the X-ray method is not infallible as commonly believed, especially if not run with the necessary attention.[24, 51]

Chiroptical techniques are a family of techniques purposely designed to analyze chiral non-racemic samples. They include the already mentioned ECD, VCD and OR, and other techniques such as optical rotatory dispersion (ORD, namely OR measured at multiple wavelengths), Raman optical activity (ROA), circularly polarized luminescence (CPL), fluorescence-detected CD (FDCD) and others.[20, 21] We would like also to mention a promising technique called light-induced Coulomb explosion imaging (CEI), suitable for AC assignment in the gas phase.[52] This and other emerging gas-phase techniques have been so far only applied to a few small test molecules.[53] This review will focus on ECD, VCD and OR/ORD methods, whose principles and applications are discussed in detail in the next sections.

The chiroptical spectra of the two enantiomers of any chiral compound are the mirror image of each other, i.e. the sequence of bands is the same in shape, position and intensity but with opposite sign. Therefore, an ECD or VCD spectrum is unique to a specific enantiomer of a given chiral substance, and if proper means of interpretation are available, it may be used to assign the AC. For example, if the $\mathrm{CD}$ of a natural compound is already known and safely related to the $\mathrm{AC}$, the comparison with the $\mathrm{CD}$ spectrum of a new sample provides the AC of this latter, in analogy with the $[\alpha]$ value. This is again a relative method, however. The biggest potential of chiroptical techniques is instead in their use as absolute methods. A survey of methods for interpreting chiroptical data is provided in section 4 .

TABLE 1: Facts for ECD/VCD/OR measurements.

\begin{tabular}{|c|c|c|c|c|c|}
\hline \multirow[b]{2}{*}{ Technique } & \multirow{2}{*}{$\begin{array}{l}\operatorname{Cost}^{(a)} \\
(1000 \\
\text { USD) }\end{array}$} & \multirow{2}{*}{$\begin{array}{l}\text { chromo- } \\
\text { phore } \\
\text { required }\end{array}$} & \multirow{2}{*}{$\begin{array}{c}\text { Sample } \\
\text { amount }^{(b)} \\
(\mathrm{mg})\end{array}$} & \multicolumn{2}{|c|}{ Interpretation } \\
\hline & & & & $\begin{array}{l}\text { Quali- } \\
\text { tative }\end{array}$ & $\begin{array}{l}\text { Compu- } \\
\text { tational }\end{array}$ \\
\hline ORD & $>25$ & no & $1-10$ & yes & yes \\
\hline ECD & $>65$ & yes & $>10^{-3}$ & yes & yes \\
\hline VCD & $>100$ & no & $>5$ & no & yes \\
\hline
\end{tabular}

(a) Average cost of the instrumentation. (b) Minimum sample amount required for measurement.

\section{SELECTED CHIROPTICAL TECHNIQUES: ECD, VCD AND OR/ORD.}

In this section we introduce the principles of the three selected chiroptical techniques, namely ECD, VCD and OR/ORD, and briefly discuss the conditions of measurements. The most relevant facts are summarized in Table 1.

\subsection{ECD: basics and measurements}

Electronic CD is defined as the differential absorption of circularly polarized radiation in the UV-Vis region of the electromagnetic spectrum, where electronic transitions occur between the electronic ground state and one or more excited states of a molecule.[54] The molar quantity $\Delta \varepsilon$ expressed in units of $\mathrm{M}^{-1} \mathrm{~cm}^{-1}$ is defined as:

$\Delta \varepsilon=\varepsilon^{L}-\varepsilon^{R}=\frac{A^{L}-A^{R}}{b c}=\frac{\Delta A}{b c}$

where $A^{L}$ and $A^{R}$ are the absorptions of left and right circularly polarized light, $c$ is the molar concentration, and $b$ is the path-length in $\mathrm{cm}$. For historical reasons the ECD is usually measured as ellipticity $\theta$ (in mdeg), connected to $\Delta A$ through $\theta=33,000 \Delta A$. The theoretical quantity associated with ECD is the rotational strength $R_{0 j}$, defined for each electronic transition from the ground state 0 to the excited state $j$ as the scalar product of electric and magnetic transition moments:[55]

$R_{0 j}=\vec{\mu}_{0 j} \cdot \overrightarrow{\mathrm{m}}_{0 j}$

The integral of the ECD band (also called Cotton effect) allied with the $0 \rightarrow j$ transition is proportional to $R_{0 j}$. The fact that the rotational strength depends on the angle between the transition dipoles makes ECD (as well as VCD and OR) more dependent on the geometry than the corresponding non-chiroptical techniques such as UV-Vis or IR spectra, which are much less sensitive toward conformation and nonsensitive toward AC.

A useful quantity related to ECD is the $g$-factor, also called the anisotropy or dissymmetry factor. The $g$-factor is defined as the ratio between ECD and absorption, and is therefore independent of the concentration and path-length:

$g_{E C D}=\frac{\Delta \varepsilon}{\varepsilon}=\frac{\Delta A}{A}$

Among the three selected chiroptical techniques, ECD is the most versatile from the viewpoint of measurement conditions. A schematic illustration of a typical ECD instrument (spectropolarimeter) is shown in Figure 2. It is very similar to a standard spectrophotometer for UV-Vis absorption measurements, except for the addition of a device, called photoelastic modulator (PEM), which produces left- and right-circularly polarized radiation alternatively. The commonly investigated range is $185-700$ $\mathrm{nm}$, however shorter wavelengths may be reached with 
special instrumentation.[56] ECD may be measured on several states of the matter, the most relevant to natural products being the solution and the solid state. Any solvent transparent in the UV-Vis region can be employed, the most useful ones being water, alcohols, acetonitrile, ethers (diethyl ether and THF), saturated hydrocarbons, chlorinated solvents $\left(\mathrm{CH}_{2} \mathrm{Cl}_{2}\right.$ above $230 \mathrm{~nm}, \mathrm{CHCl}_{3}$ above $\left.240 \mathrm{~nm}\right)$ and DMSO (above $250 \mathrm{~nm}$ ). Crystalline materials and powders may be measured as pellets after diluting with an inert salt such as $\mathrm{KCl}$ or $\mathrm{KBr}$, or with other techniques.[24] Whenever fungal metabolites or products isolated from other natural sources are concerned, special attention must be paid to the amount of sample necessary for the analysis.[57] In that respect, ECD surpasses other chiroptical methods such as OR/ORD, VCD and ROA at least when conventional instruments are employed (see Table 1). In favorable cases, a few $\mu \mathrm{g}$ of sample are required to measure a meaningful ECD spectrum. Concentration (normally around $\mathrm{mM}$ ) and path length (0.01-1 cm) are adjusted to keep the absorbance below 1 for the relevant bands. The most important requisite of ECD spectroscopy is that the compound contains at least one chromophore, i.e. a unit undergoing one or more electronic excitations in the UV-Vis range. This is not a serious limitation in the field of fungal metabolites or natural products, as the very large majority of them contains at least a $\mathrm{C}=\mathrm{C}$ or $\mathrm{C}=\mathrm{O}$ double bond. Otherwise, a so-called Cottonogenic derivative, i.e. a derivative with one or more detectable ECD bands, may be investigated.

(a)

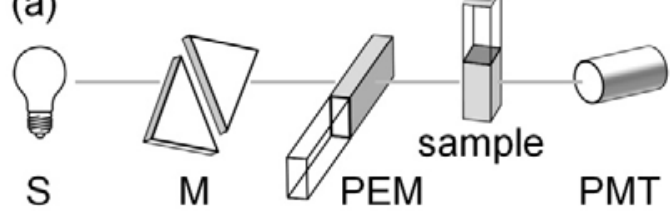

(b)

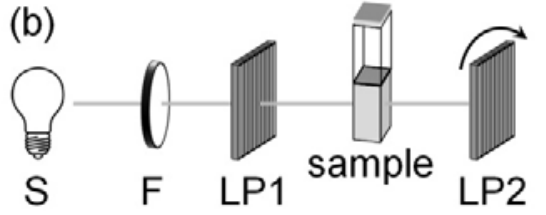

FIGURE 2: Sketch of ECD/VCD (a) and OR (b) instruments. Legend: $\mathrm{S}$, source; $\mathrm{M}$, monochromator (this is replaced by an interferometer in FT-IR); PEM, photoelastic modulator; PMT, photomultiplier; F, wavelength filter; LP1, linear polarizer; LP2, linear polarizer (analyzer).

\subsection{VCD: basics and measurements}

Similarly to ECD, vibrational CD is defined as the difference in the absorbance between left and right circularly polarized infrared radiation during a vibrational excitation. [58] The differential molar absorptivity $\Delta \varepsilon(\bar{v})$ at a given wavenumber is also expressed in $\mathrm{M}^{-1} \mathrm{~cm}^{-1}$ and defined as:
$\Delta \varepsilon(\bar{v})=\varepsilon^{L}(\bar{v})-\varepsilon^{R}(\bar{v})=\frac{A^{L}(\bar{v})-A^{R}(\bar{v})}{b c}=\frac{\Delta A(\bar{v})}{b c}$

where $A^{L}, A^{R}, c$, and $b$ are defined as said above. The intensity (integral) of the VCD band is related to the rotational strength $R_{0 v}$. For each vibrational transition, associated to a given normal mode and occurring between the zero-th $(0)$ and the $v$ vibrational sublevel of the ground electronic state, the rotational strength is defined as the scalar product of the corresponding electric and magnetic transition moments:[58]

$R_{0 v}=\vec{\mu}_{0 v} \cdot \overrightarrow{\mathrm{m}}_{0 v}$

The rotational strength sign then depends on the angle between the electric and magnetic transition moments, therefore on the overall molecular geometry. It follows that for a couple of enantiomers the same vibrational transition will have rotational strength opposite in sign.

VCD spectra can be measured in the mid-IR region $(800$ $2000 \mathrm{~cm}^{-1}$ ) by either dispersive or Fourier transform instruments.[58] Dispersive VCDs are still used for biological samples, taking advantage of a stronger source and a narrower spectral range,[59] while modern FT-VCD spectrometers[60] are commonly employed for recording spectra of small molecules and for their AC assignments. Specially designed dispersive and FT instruments are also employed for acquiring VCD spectra in the NIR (4000$14000 \mathrm{~cm}^{-1}$ ) region, populated by overtone and combination bands of fundamental vibrational modes, but NIR-VCD spectroscopy has not been employed for AC assignments so far.[61] Some differently designed FT-VCD instruments are available on the market but all of them use an infrared PEM placed in front of the sample in an FT-IR spectrometer to modulate the IR beam between left- and right-circular polarizations at high frequency (Figure 2). A number of instrumental advances have been also proposed in the last years to improve baseline stability, suppress artifacts, and provide a better signal-to-noise ratio.

Measurement of VCD is usually accompanied by measurement of IR, allowing one to identify the main vibrational transitions, and is performed either on neat liquids or in solution of a suitable solvent having a good spectral window in the IR region of interest. The best solvents are relatively non-polar and, if hydrogens are present, the deuterated analog is to be preferred, having vibrational bands shifted at lower frequency. Commonly employed solvents are $\mathrm{CDCl}_{3}, \mathrm{CD}_{3} \mathrm{CN}, \mathrm{DMSO}-d_{6}, \mathrm{CCl}_{4}$, $\mathrm{CS}_{2}$. The sample is dissolved in the solvent and placed in an IR sample cell, usually with $\mathrm{BaF}_{2}$ or $\mathrm{CaF}_{2}$ windows. In order to have a good VCD signal-to-noise ratio, concentration or path length are adjusted so that absorbance of the IR bands is in the 0.2 to 0.8 range. Compared to a typical electronic ECD signal, VCD signal is usually quite weak, about $10^{4}-10^{6}$ times lower than the intensity of the corresponding IR band. Therefore, the acquisition of a VCD 
spectrum with a good signal-to-noise ratio requires a relatively high sample concentration (about $10^{-1} \mathrm{M}$ ) and several thousands of scans (some hours of acquisition). The amount of sample required is usually in the range of milligrams (Table 1). The main advantage of VCD over ECD is that, relying on vibrational transitions, it does not require the presence of chromophores on the molecule. It follows that such technique can be potentially employed for the AC assignment of any chiral molecule, including alkanes.[62]

\subsection{OR and ORD: basics and measurements}

Chiral molecules display the property to rotate the plane of polarization of a linearly polarized incident light beam. The angle of rotation $\alpha$ depends on the radiation wavelength, the temperature, the solvent, and the sample concentration. Therefore, a more useful value to take into account is the specific rotation $[\alpha]^{T} \lambda$, also called $\mathrm{OR}$, and defined as:

$\mathrm{OR}=[\alpha]_{\lambda}^{T}=\frac{100 \alpha}{l c}$ (solution)

where $\alpha$ is the angle measured by the polarimeter (in degrees), $l$ is the path-length in $\mathrm{dm}$, and $c$ is the sample concentration in $\mathrm{g} / 100 \mathrm{~mL}$. The units for $[\alpha]_{\lambda}^{T}$ are $\operatorname{deg}[\mathrm{dm}$ $\left.\left(\mathrm{g} / \mathrm{cm}^{3}\right)\right]^{-1}$. For a neat liquid OR is defined as follows:

$\mathrm{OR}=[\alpha]_{\lambda}^{T}=\frac{\alpha}{l \rho} \quad$ (neat liquid)

where $\rho$ is the liquid density $\left(\mathrm{g} / \mathrm{cm}^{3}\right)$. When OR is measured as a function of the wavelength of light it is called ORD. For ORD the quantity called molar rotation $[\phi]$ is also used, which is the specific rotation multiplied by the molecular weight $M$ over 100 . The units of $[\phi]$ are degrees $\mathrm{cm}^{2} \mathrm{dmol}^{-1}$.

$[\phi]_{\lambda}^{T}=\frac{[\alpha]_{\lambda}^{T} M}{100}$

Such a property of chiral molecules, or generally speaking of chiral media, is due to the different refractive index $n$ of left and right circularly polarized light in those media, a phenomenon called circular birefringence and determined by the different velocity in a chiral medium of the two circularly polarized components of the linearly polarized radiation:

$\Delta n=n_{L}-n_{R} \neq 0$

where $n_{L}$ and $n_{R}$ are the refractive indices for left and right circularly polarized light, respectively. Ordinary indices of refraction are of the order of unity, and typically $\Delta n$ is only about one millionth of the absolute value of $n_{L}$ and $n_{R}$. OR at a single wavelength, such as the sodium D-line at $589.3 \mathrm{~nm}$, is commonly used to detect and quantify optical activity, whereas ORD is commonly used to determine AC. The following relationship relates the difference in the refractive indices for left and right circularly polarized light $(\Delta n)$ to the angle of rotation $\alpha$ :

$\alpha=\frac{1800}{\lambda(\mathrm{cm})}\left(n_{L}-n_{R}\right)$

Unlike ECD spectroscopy, which provides signals only in the correspondence of UV-Vis absorptions, ORD may be detected over all wavelengths. This is because ORD is based on the measurement of differences in refraction indices, and any substance exhibits a molecular refraction at almost any wavelength of the incident radiation. On the contrary, ECD involves the measurement of the differential absorption of left and right-handed circularly polarized light which occurs only in the presence of a molecular electronic transition. Hence ECD occurs only in the vicinity of an absorption band, whereas ORD is theoretically finite everywhere. This also implies that for ORD measurement the presence of a chromophore on the molecule is not strictly necessary, thus allowing to treat also UV-Vis transparent molecules.

OR is measured by a polarimeter (Figure 2) with the sample employed either neat or in solution of a suitable solvent not absorbing in the range of measurement. Commonly, $1.0 \mathrm{dm}$ path-length cylindrical quartz cells are employed and sample quantities in the range of 1-10 milligrams are required (Table 1). OR measurement at the single $589.3 \mathrm{~nm}$ wavelength is performed using a sodium lamp, while measurements at different wavelengths can be obtained by using either a mercury or a xenon lamp equipped with suitable wavelength filters. An ORD curve can be obtained either by measuring point-by-point OR at different wavelengths with a common polarimeter, or employing a spectropolarimeter, often obtained applying an ORD attachment to an ECD spectrometer. Far from absorption maxima ORD appears as an increasing (or decreasing) plain curve, but shortly before reaching the wavelength corresponding to an UV-Vis absorption maximum it reaches a maximum (peak), after which it suddenly decreases (in its absolute value) inverting its sign until another inflection point (trough) is reached. The rotation absolute value then tends to increase again. Around an absorption maximum, the ORD curve has then a typical S-shaped appearance. The smoothly rising or decreasing part of the curve is referred to as a plain dispersion curve, which can be either positive or negative, depending on the sign of the rotation values. The part of the curve in which the sign inversion is observed is called Cotton effect (CE) or anomalous dispersion. It follows that higher OR values are measured approaching the absorption maxima of the molecule.

\section{MEANS OF INTERPRETATION OF ECD, VCD AND OR/ORD}

The main topic of the present review is the quantummechanical calculation of chiroptical spectra of fungal 
metabolites, which is the modern and currently most popular way of assigning ACs based on ECD, VCD and OR/ORD data. However, especially in the context of ECD, there are several different approaches to interpret the spectra, and to establish structure/spectrum relationships which will ultimately lead to the desired piece of information - namely, the assignment of the absolute stereochemistry. In this section, we will discuss more in details the available means to interpret chiroptical data. Even for the specific problem of assigning the $\mathrm{AC}$ of a natural product, many different approaches based on ECD/VCD/OR(D) are possible, and the decision on which approach has to be followed depends on several factors such as the nature and structure of the compound (complexity, flexibility, presence of chromophores and functional groups), the amount of sample available, the ease of derivations, and so on.[63] A general concept valid for all chiroptical techniques is their dependence on the whole molecular structure, that is, both the configuration and the conformation. While the $\mathrm{AC}$ has a one-to-one correspondence with the sign, the molecular conformation affects the overall spectral shape, possibly also including the sign, in a very sensitive way.[64]

\subsection{Interpretation of ECD data}

Of the three techniques, ECD is the one with a more established tradition for the determination of $\mathrm{AC}$ of organic compounds including natural products. It is also the technique for which more different approaches are available to establish structure/spectroscopy relationships, that is, to extract the stereochemical information, such as the AC. We may recognize the following more or less broad families of approaches:

(1) comparison with a reference spectrum;

(2) empirical spectral correlations;

(3) semi-empirical rules, including for example sector rules (e.g. ketone octant rule), helicity rules (e.g. $\beta$-lactam helicity rule, chromane helicity rule), chirality rules, and others;

(4) the exciton chirality approach and other non-empirical straightforward methods;

(5) simplified calculations approaches, such as the coupled-oscillator and related methods;

(6) full quantum-mechanics calculations, by timedependent density functional theory (TDDFT) or other methods.

The first two methods are relative methods, in the sense defined in $\$ 2.3$. Method (1), already mentioned in $\$ 2.3 .2$, is the ECD equivalent of comparing $[\alpha]$ values. Method (2) consists in comparing the ECD spectrum of the target compound with those of structurally-related molecules. This kind of empirical spectral correlations have many drawbacks and their use is discouraged. As a proof, the AC of some fungal metabolites (Scheme 1), previously assigned by empirical ECD correlations, has been recently revised by applying QM ECD calculations.[65, 66]

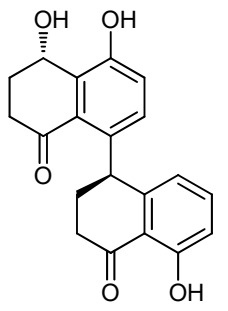

Cladosporol C from

Cladosporium cladosporioides

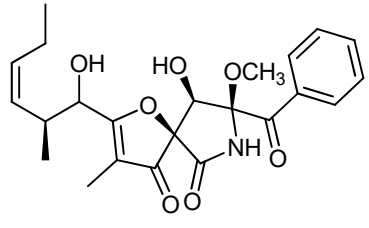

Pseurotin $\mathrm{A}_{2}$ from Aspergillus fumigatus
SCHEME 1: Structure of fungal metabolites whose AC was recently revised.

Semi-empirical rules (3) follow from the observation that a molecule may be ideally partitioned in different portions, namely chromophoric and non-chromophoric fragments. Chromophores may be further classified into intrinsically chiral (i.e., a non-planar $\beta$-lactam ring) and achiral (i.e., an aromatic ring or a carbonyl group). When a chromophore is itself or intrinsically chiral, its deviation from planarity may be defined through some helicity content. For example the $\beta$ lactam rings in penicillins, cephalosporins and related compounds are not planar. The deviation from planarity, that is, the absolute conformation of the $\beta$-lactam ring, correlates with the sign of the $n-\pi^{*}$ band of the amide chromophore around 230-260 $\mathrm{nm}$.[67] This is one example of a helicity rule. When the chromophore itself is achiral but it is comprised in a chiral molecule, the perfect symmetry of the achiral chromophore is disrupted by the surrounding chiral skeleton, whose various portions act like perturbers. The space around the achiral chromophore may be divided in sectors delimited by symmetry planes or orbital nodal planes. Two pertubers lying in enantiotopic sectors will provide, by definition, opposite contributions to any ECD band due to the chromophore. This is the essence of sector rules such as the octant rule for saturated ketones, where one observes the $n-\pi^{*}$ band of the carbonyl chromophore around 280-300 $\mathrm{nm}$. The octant rule has found many applications in the field of natural products, first of all steroids.[68] If the chromophore is achiral but embedded in a chiral cycle, for example a benzene fused to a saturated ring such as in tetralins, chromans, isochromans, tetrahydroquinolines and so on, the absolute conformation of the saturated ring dictates the ECD sign of the benzene transitions. This allows the formulation of helicity rules such as the isochroman helicity rule, related to the ${ }^{1} \mathrm{~L}_{\mathrm{b}}$ transition around $270-300$ $\mathrm{nm}$.[69] As an example of application to fungal metabolites, the rule has been applied to pseudoanguillosporin A and B extracted from Pseudoanguillospora sp, isolated from the red algae Polyides rotundus (Scheme 2).[70] In this case, the sign of the diagnostic band is related to the helicity assumed by ring $\mathrm{B}$, which is in turn dictated by the configuration at $\mathrm{C}$ 3. What all the aforementioned semi-empirical rules have in common is: a) they focus on only one or a few transitions of 
a specific chromophore, therefore their scope is intrinsically limited; b) they are semi-empirical, in the sense they depend on a collection of previous experimental data, therefore exceptions are always possible.[71] Despite these known limitations, some semi-empirical rules have been very popular in the field of natural products until the advent of modern computational techniques to be discussed below.<smiles>Cc1c(O)cc(O)c2c1C[C@H](C)OC2</smiles>

Pseudoanguillosporin A

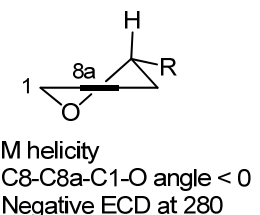

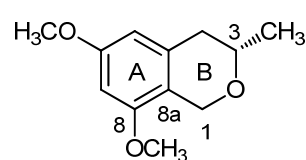

Pseudoanguillosporin B

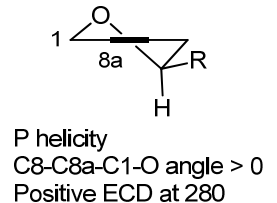

SCHEME 2: Application of the isochroman helicity rule to pseudoanguillosporin A and B.

The exciton chirality ECD (ECCD) approach (4) has been for a long time the most employed method for the AC assignment of natural products, either directly or upon preliminary chemical derivatization.[17] ECCD is applicable to molecules endowed with two or more separate chromophores giving rise to electric-dipole allowed transitions and properly arranged with respect to each other. In particular, the electric transition dipole moments should be not parallel, or coplanar, or collinear. In these conditions, the through-space coupling between the transition dipoles produces, in the ECD spectrum, a so-called ECD exciton couplet, that is, two bands of opposite sign and similar intensity, centered around the chromophore UV maximum (Figure 3). The positive or negative sign of the exciton couplet depends on the absolute angle of twist (clockwise or counter-clockwise) between the two transition dipoles, whilst the shape and intensity are sensitive to the overall arrangement (value of twist angle and interchromophoric distance). To assign the AC of a chiral organic compound, for example a novel natural product, containing two "skewed" chromophores, it is first necessary to establish the molecular conformation, that is, the relative arrangement of the two transition dipoles. Thereafter, the sign of the ECCD couplet will immediately tell the absolute sense of twist between the two transition dipoles, that is, the AC (Figure 3). Although this exciton chirality rule may sound somewhat empirical, it is not at all, because it is based on a firm theoretical basis. If a molecule does not contain two suitable chromophores, one or two chromophores may be introduced by linking them at appropriate functional groups such as alcohol, amine, carboxylic acid and so on (see the example in Scheme 3). Standard microscale derivatization/analysis procedures exist for various classes of substrates, which encompass many structural motifs found in natural compounds, for example 1,2-diols, polyols, aminoalcohols, etc. $[72,73]$<smiles>O=C1CC[C@@H](O)[C@@]23OC4(Oc5cccc6cccc(c56)O4)C12C1O[C@@H]1C3O</smiles>

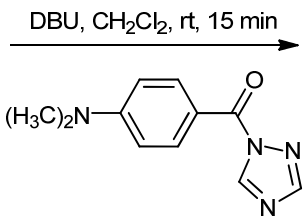

SCHEME 3: Conversion of diepoxin $\eta(\mathbf{1})$ in its $\operatorname{bis}(p-$ dimethylaminobenzoate) 1a suitable for ECCD analysis.

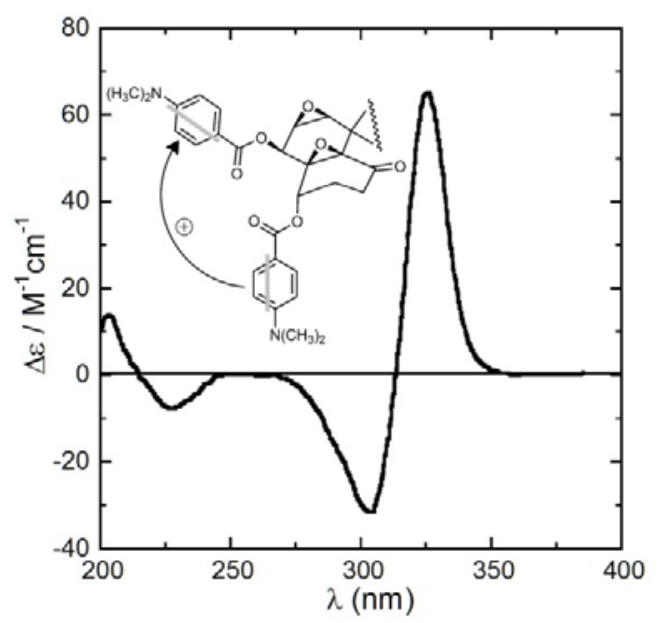

FIGURE 3: Exciton coupling in the ECD spectrum of diepoxin $\eta$ bis ( $p$-dimethylaminobenzoate) 1a and application of the exciton chirality rule. Courtesy of Nina Berova.

Alternatively, methods based on supramolecular chemistry have been developed where an achiral chromophoric host interacts with a chiral guest and acquires a diagnostic ECCD spectrum which reports the guest chirality.[74] The most notable example is the bis-porphyrin tweezers method introduced by Berova, Nakanishi and coworkers, where the chiral guest is obtained by functionalization of target molecules with a single site of derivatization.[73, 74] The above mentioned approaches based on ECCD have been employed in hundreds of cases including natural products, for which we refer the readers to the Harada and Nakanishi book and several recent reviews.[17, 72, 73] An example of the exciton chirality method applied to a fungal metabolite is shown in Figure 3. Here, diepoxin $\eta$ (1), a spiroketal naphthoepoxydecalinone of fungal origin endowed with antitumoral activity, was converted to its 4,5-bis ( $p$-dimethylaminobenzoate) 1 a by the procedure shown in Scheme 3.[75] The $p$ - 
dimethylaminobenzoate chromophore has a strong absorption band at $315 \mathrm{~nm}$ related to the $\pi-\pi^{*}$ transition polarized along the chromophore main axis (see gray lines in Figure 3).[17] The strong positive couplet found in the ECD spectrum of 1a (Figure 3) reveals a positive chirality between the two transition moments which is in turn related to a $(4 S, 5 S)$ configuration.[75] Exciton-coupled ECD spectra may also be quantitatively calculated by means of so-called coupled oscillator approach (DeVoe) and related methods (5).[76, 77]

Apart from the exciton-chirality method, several other approaches exists of the type (4) which conjugate a nonempirical nature with ease of application, that is, they do not require any calculation.[63] The choice of these methods is made on a case-by-case basis, depending first of all on the molecular structure of the target molecule. A typical approach of this type is the use of the so-called "chiroptical probes" i.e. achiral or racemic, then ECD silent, chromophoric moieties which, when linked to an achiral substrate, give rise to specific diagnostic ECD bands. The sign of these latter is related in a non-empirical way with the AC of the investigated substrate.[78] Examples of these type are flexible biphenyls, employed as chiroptical probes to determine the AC of chiral diols and carboxylic acids.[79, 80]

The last approach (7) to be mentioned for the application of ECD spectroscopy is the focus of the present review, namely the simulation of ECD spectra by means of QM calculations.[21, 81-85] This option has become fully practicable for relatively complex molecules such as natural products only relatively recently, in parallel with the development of computer technology. The same applies to the other chiroptical spectroscopies (VCD and OR/ORD) discussed below, although the calculation times are usually longer for ECD than for VCD and OR/ORD. This is because for ECD it is necessary to explicitly calculate electronic excited states, therefore it is mandatory to use an accurate but not too demanding level of calculation. A breakthrough in this context has been represented by time-dependent density functional theory (TDDFT),[86] currently the method with the best cost/efficiency compromise. A TDDFT ECD calculation of a medium-size molecule is nowadays possible on a desktop PC in a reasonable time. A detailed description of the typical application of QM calculations of $\mathrm{ECD}, \mathrm{VCD}$ and OR/ORD is given in section 5, whilst in section 6 we will provide several examples of $\mathrm{AC}$ assignments of fungal metabolites.

\subsection{Interpretation of VCD data}

VCD spectroscopy is a much more recent technique than ECD. The first VCD measurement was reported only in 1974,[87] more than a century after the discovery of ORD and ECD. Contrary to ECD spectra and ORD curves, VCD spectra appear quite complex, displaying, like a common IR, many narrow bands in a relatively short wavenumber range (Figure 1). This makes impossible to carry out empirical correlations or define semi-empirical rules like in ECD and ORD.

The first attempt to define a non-empirical model to interpret VCD spectra was based on the application of the coupled oscillator model, analogous to the exciton chirality approach described above for ECD spectra.[88] In this case VCD arises from two identical oscillators in the molecule separated by a fixed distance and skewed relative to one another. The two oscillators couple with each other such that two new vibrational modes ensue from their coupling. The model predicts VCD signals of equal magnitude and opposite sign for the two coupled modes. This model, although fairly general, is restricted to cases where two nearidentical coupled vibrational motions can be found in a molecule. Typically such situation is encountered in dicarbonyl compounds in which the two groups are near in space and in a fixed relative disposition. [89, 90]

The most general and widely employed approach for AC assignments through VCD spectroscopy is, by far, the DFT quantum-mechanical calculation of VCD spectra.[81, 91] The first ab initio VCD calculation dates back to 1985[92] and some years later the implementation of DFT[93] provided the practical computational approach most used till now. The AC assignment is achieved by comparing the sign and intensity of the measured VCD spectrum with the DFT calculated one for a given configuration, as explained in section 5.1 below. Noteworthy, the timescale of vibrational transitions is short enough to allow the detection in the VCD spectrum of signals allied to single conformers, then making such technique particularly suitable to carry out also conformational analyses.

\subsection{Interpretation of $\mathrm{OR} / \mathrm{ORD}$ data}

OR has been for a long time the only chiroptical property employed for establishing the AC of known molecules, by simple comparison of OR sign with literature data (a relative method mentioned in \$2.3.1). Several empirical and semi-empirical methods have also been proposed, over the years, to use OR and ORD to assign the AC of unknown molecules (an absolute method mentioned in $\$ 2.3 .2$ ). The first attempt was reported by Kuhn in 1938, [94] but the more reliable and most widely employed approach was the already mentioned octant rule developed by Djerassi and co-workers[95] for the AC assignment of chiral saturated cyclic ketones by the sign of the ORD allied to the $n-\pi^{*}$ carbonyl transition.[68] From the experimental point of view, ECD has to be preferred over ORD. In fact, ECD bands occur only in correspondence of optically active absorption bands, while the ORD spectrum results from the sum of contributions from all the chromophores of the molecule, even those absorbing in the far UV. It follows that in ORD it is difficult to separate the contributions from 
different electronic transitions, while in ECD overlaps occur only between very close absorptions, thus making the spectrum much easier to be interpreted. This limitation, on the contrary, turns out to be an advantage of ORD over ECD when dealing with compounds devoid of chromophores absorbing above $180 \mathrm{~nm}$. These compounds are in fact ECD silent, while they will still show an ORD curve even at long wavelengths.

The use of OR and ORD for AC assignments has experienced a renewed interest with the advent of computational methods allowing the prediction of OR values or ORD curves of chiral molecules. The first $a b$ initio OR calculation was reported in the 1980s,[96] but only in 1997 it started to be applied to predict the ORs of chiral molecules.[97] Before the availability of dynamic (frequency-dependent) linear response methods, the static limit was used for OR computations, however, this approach is now avoided because it provides values which disagree with the correct dynamic values.[81] A few years later, the DFT implementation finally provided the QM protocol currently employed for OR predictions.[98, 99] For the AC assignment, TDDFT calculations of OR at different wavelengths (i.e. ORD) provide more reliable results, because approaching the absorption maxima the OR values become larger, thus increasing the prediction reliability.[100] In fact, according to some authors, a reliable AC assignment at a single wavelength can be obtained only for OR values greater than 30-40 units.[99]

\section{QUANTUM-MECHANICAL CALCULATIONS OF CHIROPTICAL PROPERTIES}

The QM calculation of ECD, VCD and OR/ORD spectra for the assignment of $\mathrm{AC}$ relies on a well-established procedure which is consistent and very similar for the three methods. Therefore, we may sketch a typical computational flowchart (Figure 4) which applies to all of them, with some specific issues and some minor differences which will be highlighted below. The existence of this consolidated and standardized procedure, and the fact that these calculations are now available in various software packages, have encouraged many non-experts to use the QM approach to establish the AC of many compounds, including natural products. However, a black-box usage of computational tools may easily lead to wrong results if not properly faced. The following section, apart from giving an overview of the methods actually used in most literature examples, also aims to guide the reader through a good computational practice in QM calculations of chiroptical spectra.[85]

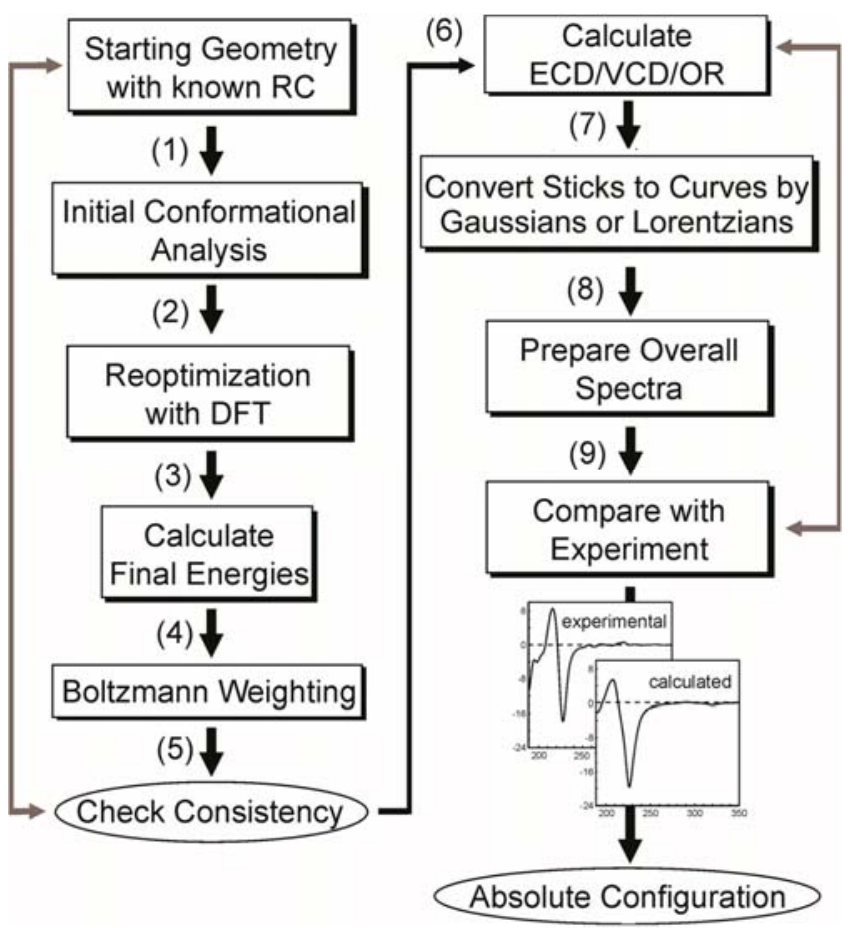

FIGURE 4: Flowchart showing the typical steps in QM calculations of $\mathrm{ECD} / \mathrm{VCD} / \mathrm{OR}$.

\subsection{A general computational flowchart for ECD/VCD/OR calculations}

The starting point of the computational procedure is the input structure of the target molecule. For fungal metabolites the structure is normally elucidated by means of the spectroscopic methods summarized in section 2 . We assume that the structure has been elucidated in all its aspects, including the $\mathrm{RC}$ of the various chirality elements, and the only missing piece of information is the AC. In principle, the $\mathrm{RC}$ may also be determined by chiroptical spectroscopies, especially by employing a combination of ECD and VCD.[101, 102] However, we recommend the use of the independent tools discussed in $\$ 2.2$ to establish the RC. The input structure with the correct constitution and $\mathrm{RC}$ will then be generated by a molecular modeling software with an initially arbitrary AC. The typical steps of the QM procedure are the following (Figure 4).

(1) Perform a thorough conformational search using molecular mechanics (MM) with a good force field (FF), for example MMFF (Merck Molecular FF),[103] to sample the molecular conformation.[104] In fact, any molecular property is an average over the whole sets of conformations which may be adopted by the molecule at the working temperature. The more large and flexible is the molecule, the more structures will be found at this stage. For flexible molecules it is recommended to use a conformational analysis protocol such as Monte Carlo or molecular 
dynamics.[105] Although this procedure is largely automatized, it is important to check that all rotatable bonds are varied and all atoms included in cycles are puckered, in order not to miss any possible conformer.[106]

(2) Run the geometry optimization of all conformers found in step (1) using density functional theory (DFT). This is typically done using the B3LYP/6-31G(d) combination, however other functionals and basis sets may perform better. $[85,107]$ As for the functional, recommended ones are M06$2 \mathrm{X}$ and $\omega \mathrm{B} 97 \mathrm{X}-\mathrm{D}$ (which also includes a dispersion correction). As for the basis sets, Ahlrichs' basis sets such as def2-SVP or def2-TZVP are better suited than Pople's family (e.g. 6-31G(d) and following). After this step, a restricted number of low-energy conformations is kept, falling in an energy window within a certain threshold, i.e. 5 or $10 \mathrm{kcal} / \mathrm{mol}$. Thereafter, one may run a second optimization with a more refined method, for example using a larger basis set with additional polarization or diffuse functions $(6-311 \mathrm{G}+(\mathrm{d}, \mathrm{p})$ or def2-TZVP), or including a continuum solvent model.[108]

(3) The final set of low-energy conformations obtained from point (2) is ordered according to their internal or free energies (see point 6), where the lowest-energy conformer is taken as zero-energy reference. One should then decide how many conformers need to be considered for the next calculations, by applying an energy threshold. Normally, all conformers with relative energy above 2.5 or $3 \mathrm{kcal} / \mathrm{mol}$ may be disregarded.

(4) Apply the Boltzmann distribution to the set of selected low-energy minima obtained in step (3), by using internal or free energies. A final set of structures is thus obtained together with their respective Boltzmann populations at the working temperature $(298 \mathrm{~K}$ or $300 \mathrm{~K})$. All conformers with Boltzmann population above some threshold, say $1 \%$, are kept for the following steps.

(5) Check the experimental consistency of the calculated conformational ensemble with the available NMR data (Jcouplings, NOE contacts, ring-current shifts). We consider this step absolutely necessary in any well-done conformational analysis.[104] Unfortunately, it is very often overlooked especially by non-specialists who tend to be too confident in computational results. Moreover, this is the stage at which one may further test the reliability of the input structure. If the most stable calculated conformers are contradicted by experimental data, this is a possible hint of a wrong initial structure, either the constitution or the RC. As already noted in $\$ 2.2$, flexible molecules are always a challenge for the correct interpretation of NOE data. In this situation one may resort to low-temperature NMR experiments, which in favorable cases also allow a direct quantification of conformers and highlight stereodynamic processes.[104, 109]
(6) Calculate the desired chiroptical property for all relevant conformers. This is the most important step in the whole procedure, and the method to be used depends on the specific calculation, either ECD, VCD or OR/ORD. For ECD calculations, one needs to evaluate electronic excited states and TDDFT is, as said above, the method of choice for most common situations. Electronic absorption UV-Vis spectra are also calculated in parallel with ECD. In these calculations, the number of excited states (roots) must be decided; this is chosen in a way to cover the whole experimentally available range, and extend well below the lowest observed wavelength. For $V C D$ calculations, one must run frequency calculations at the same level used for geometry optimizations, and DFT is the method of choice. IR spectra are calculated in parallel with VCD. The number of normal modes is automatically selected by the software, and covers all possible observed IR/VCD bands. For OR/ORD calculations, DFT is again preferentially employed at least for medium-sized molecules such as fungal metabolites. OR calculations are run for one or more discrete wavelengths (always including the sodium D-line, e.g. 589.3 $\mathrm{nm})$. ORD calculations are in fact just OR calculations run for three or more such wavelengths, usually in the Vis range. In all cases, the choice of the level of calculation is very crucial. As far as DFT and TDDFT calculations are concerned, this means that a careful choice of the functional and the basis set is mandatory. The most important advice here is that one should never be satisfied only with a single combination (one functional and one basis set): on the contrary, multiple combinations with different functionals and basis sets should be explored.

As for the functional, the popular B3LYP is in fact often the best choice for VCD calculations. However, this is seldom true for ECD and possibly OR/ORD calculations. In these latter cases, the two families of hybrid functionals and range-separated functionals should be considered. Within hybrid functionals, a crucial parameter is the fraction of "exact" or Hartree-Fock (HF) exchange. Popular hybrid functionals listed in order of increasing HF exchange are: B3LYP (20\% HF); PBE0 (25\%); M06 (27\%); BH\&HLYP (or BHLYP, 50\%); and M06-2X (54\%). Range-separated functionals use an increasing amount of HF exchange (higher at long range), and are designed to alleviate the typical problem of incorrect asymptotic behavior.[110, 111] Some well-performing range-separated functionals are CAM-B3LYP, $\omega$ B97X and $\omega$ B97X-D.

As for the basis set, basis sets of double or triple- $\zeta$ quality, with a sufficiently wide set of polarization functions, are recommended. Diffuse functions may be necessary for ECD calculations when Rydberg states contribute substantially to the ECD spectrum,[112] or for OR calculations of small molecules.[98, 99] The choice of the basis set is in general less crucial for ECD than for VCD and OR/ORD. In all cases, a simple pragmatic approach may help finding the correct basis set for any calculation. This 
consists in running several DFT or TDDFT calculations on one representative conformer keeping the functional fixed and increasing the basis set size within one family. For example, one may explore, in increasing order: SVP, TZVP, minimally-augmented TZVP and fully-augmented TZVP (aug-TZVP), from the Ahlrich's family; or 6-31G(d), 6$31 \mathrm{G}(\mathrm{d}, \mathrm{p}), \quad 6-31+\mathrm{G}(\mathrm{d}), \quad 6-311+\mathrm{G}(\mathrm{d}, \mathrm{p})$, and $6-311++\mathrm{G}(\mathrm{d}, \mathrm{p})$, from the Pople's family. Then, one looks at the basis set convergence, i.e. selects the smallest basis set giving results comparable with the larger ones. In other words, the best cost-accuracy compromise is looked for.

All chiroptical data are solvent-dependent,[113] therefore in this step the use of at least a continuum solvent model is beneficial.[108] In rare cases, however, only the inclusion of explicit solvent molecules leads to a satisfying calculation result. Generally speaking, VCD and OR/ORD data are more sensitive to solvent than ECD, both from an experimental and a computational viewpoint.

All other issues specific to each of the techniques considered will be discussed in section 5.2.

(7) ECD and VCD calculations provide a list of rotational strengths as a function of wavelength or wavenumber, that is, a stick plot. To generate true ECD and VCD spectra one must apply a band-shape to each rotational strength and sum over the whole spectral range. A Gaussian band-shape is normally used for ECD,[114] with a band-width (the standard deviation of the Gaussian curve) chosen on a bestfit basis; reasonable values for the FWHM (full width at half maximum) or the exponential bandwidth $\sigma$ are in the range $0.15-0.3 \mathrm{eV}$. A Lorentzian band-shape is instead normally used for VCD, with FWHM of 4 or $8 \mathrm{~cm}^{-1}$. The same bandshape is applied to the calculated absorption UV-Vis and IR spectra, respectively. This is not necessary for $\mathrm{OR}$ calculations, because OR and ORD data are usually discretized.

(8) Calculate the final Boltzmann-averaged ECD or VCD spectrum, or OR values. This is achieved first by weighting each component spectrum or value obtained in step (7) with the Boltzmann weights estimated in step (4), and then by adding all weighted spectra or values to each other. In the Boltzmann distribution, one may use internal energies or, especially for VCD calculations, zero-point corrected energies or free energies (obtained in frequency calculations). In principle, free energies are more accurate than internal energies, because the former include the vibrational entropic factors. In practice, however, the calculated entropies may be largely inaccurate for lowenergy vibrations,[115] so that internal energies are more commonly employed.

(9) Compare the experimental ECD/VCD/ORD spectrum with the final calculated ECD/VCD/ORD spectrum over the whole available spectral range. The simultaneous comparison of UV-Vis and IR spectra should be made. Some authors recommend to compare experimental and calculated $g$-factors instead of ECD/UV-Vis and VCD/IR spectra separately.[116, 117] In TDDFT ECD calculations, transition energies are often overestimated; this may be taken into account by applying a "manual" red-shift correction to the calculated spectra (sometimes called UV correction). In DFT VCD calculations, frequencies are always overestimated because of the harmonic approximation; this is taken into account by applying a frequency scale factor, usually between 0.98 and 0.99 . The comparison is then run after scaling the spectra. If a good match is observed between the calculation and the experiment, including the sign of all or most important bands, the assumed AC is the correct one; if instead there is a good mirror-image relationship, the opposite $\mathrm{AC}$ is correct. The spectral comparison may be run quantitatively by available tools.[21, 117, 118] If the comparison is poor for both possible enantiomers over the available spectral range, this is the signal that something went wrong, and the computational procedure should be reconsidered, first by changing and/or improving the level of calculation at step (6), and, if necessary, by starting over from step (1).

\subsection{Specific calculation issues for ECD, VCD and OR/ORD}

\subsubsection{ECD:}

As said above, a correct choice of the functional is especially important for ECD calculations. Both the wavelengths and intensities of UV-Vis and ECD bands depend on the functional employed, in particular the fraction of HF exchange and the range separation.[110, 111] There is no simple recipe for choosing the "best" functional for a given molecule, therefore the trial-and-error procedure described in $\$ 5.1$ should be tested using as many functionals as possible, and then looking at the best agreement with the experimental spectra. Two functionals which have been emerged in the last years to perform especially better in ECD calculations of organic molecules are CAM-B3LYP and $\omega B 97 X-D .[85]$ The "first-choice" B3LYP functional may perform well in many cases, but be much less satisfying in other cases, especially in the presence of aromatic chromophores undergoing charge-transfer transitions.[119]

The impact of solvent on ECD spectroscopy is usually less dramatic than for other chiroptical spectroscopies such as VCD and OR/ORD. The inclusion of a continuum solvent effect such as the polarizable continuum model (PCM)[108] will hardly reverse an $\mathrm{AC}$ assignment, however it often improves the agreement with the experimental spectrum, especially by providing a better spacing between the calculated ECD bands.

Although TDDFT is nowadays the method of choice for ECD calculations, at least in two circumstances one should consider alternative methods. These are: a) the molecule is too big and complex for TDDFT; b) the ECD spectra are dominated by charge-transfer excitations. In case a), a concrete alternative is the use of simplified TDDFT 
(sTDDFT), a very efficient methods which allows ECD calculations of molecules with up to 1000 atoms.[120] In case $b$ ), one must resort to more time-consuming methods such as those based on coupled cluster (CC) theory.[121]

\subsubsection{VCD:}

Vibrational transitions are calculated for the electronic ground state, therefore the theoretical simulation of the VCD spectrum is carried out at DFT level and does not require more sophisticated calculations of excited states, resulting less computationally demanding than ECD. Moreover, the frequency calculation is carried out during the geometry optimizations, furnishing at the same time geometry, internal and free energy, IR, and VCD spectra for a single conformer. For VCD calculations the B3LYP functional is the most usually employed one and quite often the $6-31 \mathrm{G}(\mathrm{d})$ or TZVP basis sets are sufficient to obtain reliable results. As reported above, any VCD experimental spectrum results from the superimposition of the spectra of individual conformers, weighted by the corresponding populations. The relative small bandwidth makes also possible to sort out in the spectrum the bands allied to the single conformers. For this reason it is extremely important to carry out an accurate conformational search and geometry optimizations to obtain a good spectral simulation. Moreover, VCD is strongly affected by solvent effects, determining both conformers populations and vibrational energies. It follows that for a good spectral reproduction either implicit continuum solvent model [108] or explicit models have to be taken into account in calculations.

\subsubsection{OR/ORD:}

There seems to be a general agreement on the computational requirements needed for reliable $O R$ predictions, that is, the use of a dynamic method together with a proper treatment of the electron correlation and the use of large basis sets containing diffuse functions.[99, 122] Commonly, B3LYP and PBE0 functionals, joined with augcc-pVDZ basis set, are the method of choice for DFT OR calculations. In many cases PBE0/aug-cc-pVDZ yields a somewhat better agreement with liquid-phase experimental data than B3LYP, while in general the increase in the HF exchange contribution (e.g. BHLYP) leads to a worst agreement with experiment. For organic molecules, rangeseparated functionals do on average not outperform the established B3LYP/aug-cc-pVDZ protocol.[123] Benchmark calculations of OR(D) require higher levels of theory such as coupled cluster (CCSD). Obviously, there is a number of factors that determine the quality of agreement between computations and experiment, apart from the choice of the functional and the basis set. They include vibrational corrections, solvent effects, and the quality of optimized molecular geometries. Moreover, it must be reminded that there are also possible sources of errors affecting OR experimental data, including sample purity, concentration, volume, and temperature, which can easily change OR values up to several percent.

\section{APPLICATION OF ECD/VCD/OR CALCULATIONS TO THE ASSIGNMENT OF THE ABSOLUTE CONFIGURATION OF FUNGAL METABOLITES}

As already mentioned in the Introduction, the use of QM simulations of $\mathrm{ECD} / \mathrm{VCD} / \mathrm{OR}$ spectra as a means to assign the $\mathrm{AC}$ of natural products has been already reviewed several times.[23-34] Here, we will focus in particular on fungal metabolites, however even in this more limited context the examples from the literature are hundreds, especially from 2010 on. In the Supplementary Material (Table S1), we list all literature records we retrieved from the SciFinder database in August 2016,[124] concerning AC determinations by QM calculations of ECD, VCD or OR/ORD, which make explicit reference to fungi. For each compound (or family of strictly analogous compounds) we list in Table S1 the given name, the source fungus, the host organism, the structural class (according to the DNP),[46] the technique(s) employed for the $\mathrm{AC}$ assignment, and the literature reference. A summary of our dataset is shown in Figure 5. The rapid increase in the publications from 20092010 on is impressive. All the major structural classes of natural compounds[46] are well represented in the dataset, apart from those with more or less obvious AC (for example peptides and steroids). The most populated families are polyketides, terpenoids, alkaloids, and (hetero)aromatic compounds. From our dataset (Figure 5) it appears that the most employed single technique is by far ECD, either alone or in combination with other techniques. VCD alone was employed only in a few cases, and the same is true for OR/ORD. Finally, in a minor number of cases the three techniques were used concurrently. It is obvious, and it has been argued, that a simultaneous use of different chiroptical technique increases the reliability of any $\mathrm{AC}$ assignment.[125] On the other hand, the confidence in AC assignments by ECD or VCD is often high enough that a single assignment by either ECD or VCD will suffice. There are however some situations where the simultaneous use of ECD, VCD and OR/ORD is especially beneficial, including: a) weak experimental spectra; b) non-robust calculated spectra; c) incomplete agreement between experimental and calculated spectra. Some examples will be given below.

Because of the extension of our dataset (Table S1 and Figure 5), it is impossible to discuss all or even most literature examples in details. In the following sections, therefore, we will analyze only a few selected cases, divided according to the main technique employed. A sharp selection of examples is justified by the fact that the very large majority of literature reports are based on a consistent approach, that is, the same computational flowchart outlined above, making most cases similar to each other. 


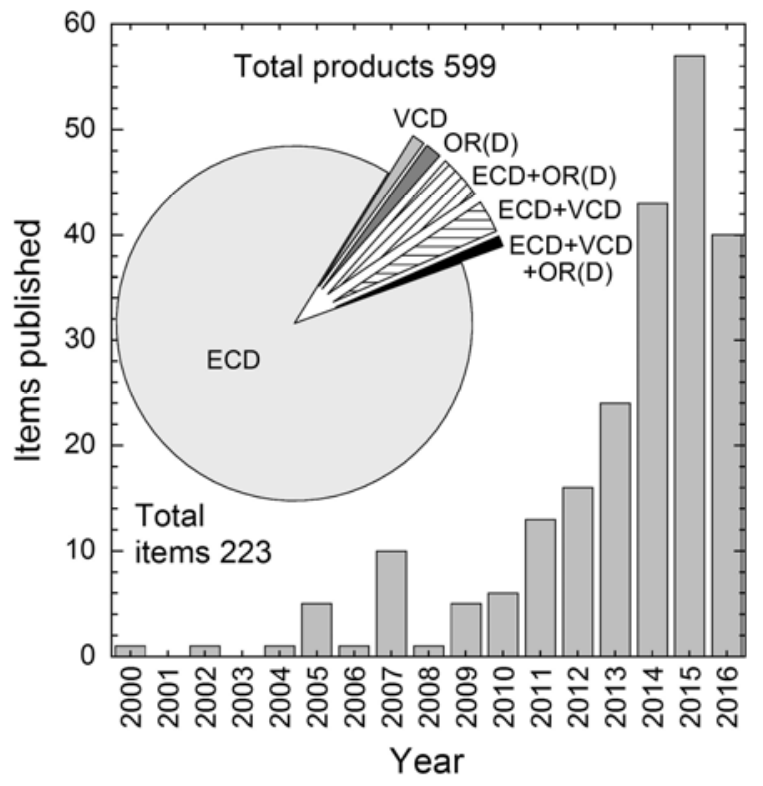

FIGURE 5: Summary of dataset about QM calculations of $\mathrm{ECD} / \mathrm{VCD} / \mathrm{OR}$ of fungal natural products retrieved from SciFinder database in August 2016.[124] The histogram refers to the publications (items published), the pie chart to the technique employed for the AC assignment.

\subsection{ECD}

As a first and typical example of the application of QM ECD calculations to fungal metabolites, we illustrate the case of a family of pyrenocine derivatives (oxygen heterocycles), called phomopsinones A-D (Scheme 4), extracted from an endophytic strain of Phomopsis sp. isolated from the halotolerant plant Santolina chamaecyparissus (cotton lavender) from Sardinia. These compounds showed antifungal, antialgal and antibacterial activity.[126] Despite the relatively simple skeleton, the assignment of the $\mathrm{RC}$ of the epimeric phomopsinones $\mathrm{B}(\mathbf{3})$ and $\mathrm{C}$ (epi-3) was not immediate. Because of the flexibility of the 2-hydroxypropyl chain, ROESY data needed to be interpreted with the help of molecular modeling. In particular, a strong NOE was observed between the pseudoaxial $\mathrm{H} 8$ and $\mathrm{H} 10$ hydrogens. While this is hardly understood looking only at the planar structures (Scheme 4), it was easily justified by inspecting the molecular models. Only for the $\left(7 S^{*}, 10 R^{*}\right)-\mathbf{3}$ isomer in fact the two hydrogens involved in the diagnostic NOE lie at short distances for one lowenergy conformer, while this is not possible for the $\left(7 S^{*}, 10 S^{*}\right)$-epi-3 isomer (Figure 6). The AC assignment of 2, 3 and 4 was obtained by the standard computational approach described in $\$ 5.1$, using MMFF conformational searches, DFT geometry optimizations at B3LYP/6-31G(d) level, and TDDFT ECD calculations run with several functionals (B3LYP, CAM-B3LYP, PBE0) and basis sets
(SVP, TZVP, aug-TZVP). The best performing functional was CAM-B3LYP, while the basis set convergence was observed already with the smallest basis set SVP. Several low-energy conformers were obtained by DFT optimizations within a $2 \mathrm{kcal} / \mathrm{mol}$ energy window in each case: six conformers for $\mathbf{2}$, ten for $\mathbf{3}$, and ten for $\mathbf{4}$. The most stable structures are shown in Figure 6. Experimental ECD spectra recorded in acetonitrile and TDDFT-calculated ECD spectra (at CAM-B3LYP/SVP level) are shown in Figure 7 for phomopsinone A (2) and D (4). The vertical bars in Figure 7 represent the calculated rotational strengths for the lowestenergy conformer in each case, that is, the "stick plot" mentioned in $\$ 5.1$, point (7). The stick plot helps visualizing the number of transitions (and their respective ECD sign and intensity) contributing to each apparent ECD band. The transitions may be then analyzed in terms of contributing excitations and orbitals. To obtain the full ECD curves, a Gaussian band-shape was applied in this case with an exponential bandwidth $\sigma=0.3 \mathrm{eV}$. Calculated spectra were red-shifted by $15 \mathrm{~nm}$ to take into account the systematic energy overestimation by CAM-B3LYP; this is the "UV correction" mentioned in $\S 5.1$, point (9). Additionally, the calculated spectrum for 2 was scaled by a factor of 1.5 to better compare with the experiment. This is also a common procedure in ECD calculations, because the calculated intensities are often overestimated both for UV-Vis and ECD spectra. In all cases, the calculated spectra matched the experimental ones quite fairly, and the established absolute configurations were $(S)-\mathbf{2},(7 S, 10 R)-\mathbf{3}$, and $(7 S, 8 R)-\mathbf{4}$.[126] From the inspection of Figure 7 , one may argue that the agreement is better for 2 than for $\mathbf{4}$. This is well appreciated by means of a quantitative spectrum comparison, for example by evaluating the so-called similarity factor (SF) with the freeware tool SpecDis.[118] The SF is comprised between 0 and 1 , and a value close to 1 indicates a perfect agreement. For compound 2, the estimated SF is 0.96 for the correct enantiomer $(S)-\mathbf{2}$, and 0.004 for the wrong enantiomer $(R)-\mathbf{2}$. For compound $\mathbf{4}$, the estimated SF is 0.83 for the correct enantiomer $(7 S, 8 R)-4$, and 0.04 for the wrong enantiomer $(7 R, 8 S)-4$. Thus, the $\mathrm{AC}$ assignment is definitely safe for both compounds.

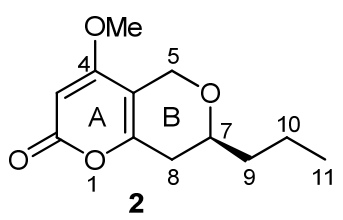<smiles>COc1cc(=O)oc2c1CO[C@H](C[C@H](C)O)C2</smiles><smiles>COc1cc(=O)oc2c1CO[C@H](C[C@H](C)O)[C@H]2O</smiles><smiles>CCC[C@H]1OCc2c(OC)cc(=O)oc2[C@H]1O</smiles>

SCHEME 4: Phomopsinones A-D extracted from Phomopsis sp.. 


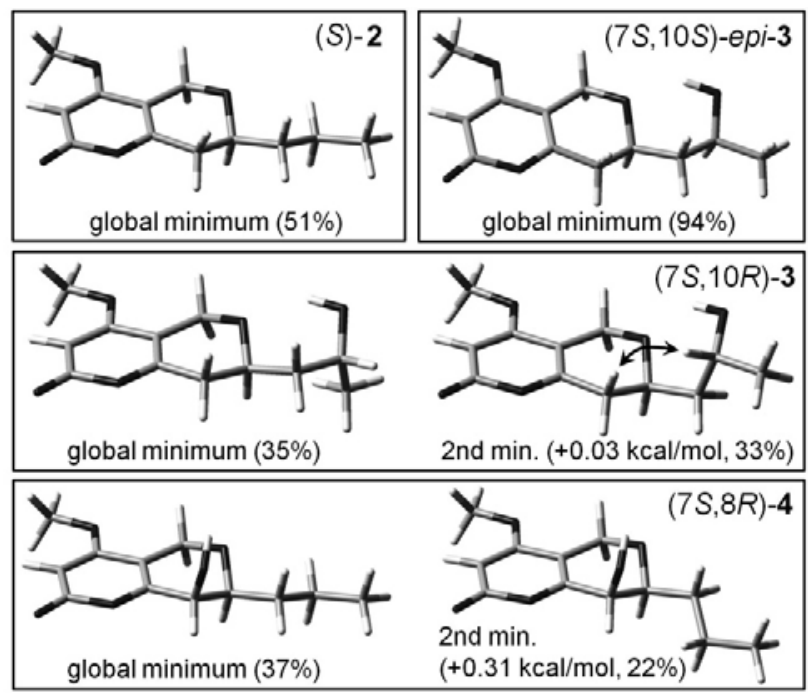

FIGURE 6: Lowest-energy DFT structures of phomopsinones A-D (2, 3, epi-3 and 4). One or two conformers summing to an overall population of at least $50 \%$ are shown, with relative energies and Boltzmann populations at $300 \mathrm{~K}$. The double arrow indicates the diagnostic NOE discussed in the text. Adapted from ref. [126], with permission.
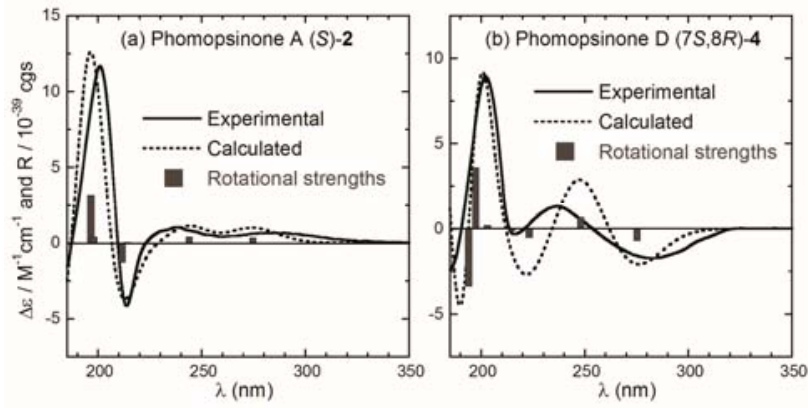

FIGURE 7: Experimental and calculated ECD spectra for phomopsinones A (S)-2 (a) and D (7S,8R)-4 (b). Experimental spectra measured in acetonitrile solution. Calculated spectra (CAM-B3LYP/SVP level) are the Boltzmann averages over six conformers for $\mathbf{2}$ and ten conformers for 4. Vertical bars represent calculated rotational strengths for the lowest-energy conformer in each case; a Gaussian band-shape with exponential band-width $\sigma=0.3 \mathrm{eV}$ was applied. Adapted from ref. [126], with permission.

A very instructive example of the possible traps in the use of ECD calculations is offered by a family of 12-membered macrolides (polyketides), named dendrodolides (Scheme 5), extracted from the fungus Dendrodochium sp. isolated from the sea cucumber Holothuria nobilis Selenka from the South China Sea. Some of these compounds exhibited cytotoxic activity toward the SMMC-7721 and HCT116 tumor cell lines.[127] The main structural feature of dendrodolides (Scheme 5) is the pronounced flexibility of the macrocycle, which not only posed a serious difficulty in the structure elucidation, but also had strong effects on ECD spectra and on their calculations. The assignment of the RC and AC of these compounds required the concurrent use of several techniques. In particular, the results of the conformational analyses, geometry optimizations and ECD calculations demonstrated that an apparently minor structural difference may induce significant conformational changes in the macrolide ring, which are reflected in the ECD spectra. This crucial aspect will be illustrated focusing our attention on the four dendrodolides A-D (5-8, Scheme 5).

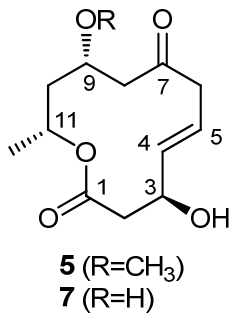

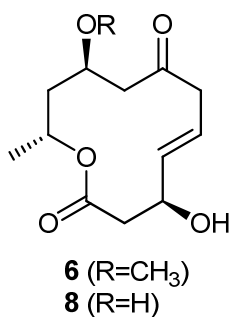

SCHEME 5: Dendrodolides A-D (5-8) extracted from Dendrodochium sp.
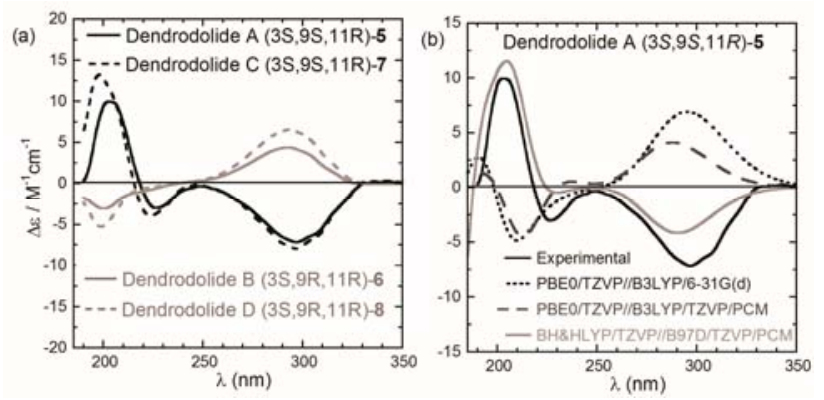

FIGURE 8: (a) Experimental ECD spectra of dendrodolides A-D (5-8) measured in acetonitrile solutions. (b) ECD spectra calculated at various levels for $(3 S, 9 S, 11 R)$ dendrodolide A (5) compared with the experimental spectrum. Calculated spectra are Boltzmann-weighted averages over the number of conformers reported in Table 2. "PCM" indicates that a solvent model for acetonitrile was used in the geometry optimizations. ECD spectra were generated as sums of Gaussians with $F W H M=0.35 \mathrm{eV}$. Adapted with permission from [127]. Copyright 2013 American Chemical Society.

The solution ECD spectra of dendrodolides A and C (5 and 7) were very similar to each other, as were those of dendrodolides B and D (6 and 8) (Figure 8). Conversely, the ECD spectra of the pairs of epimers at C-9 (namely, 5 vs 6 , and 7 vs 8), have opposite signs in the regions of both the carbonyl $\mathrm{n}-\pi^{*}$ transition around $300 \mathrm{~nm}$, and the carbonyl/alkene $\pi-\pi^{*}$ transitions around $200 \mathrm{~nm}$. This observation proves that the configuration at $\mathrm{C} 9$ is the major responsible for the appearance of ECD spectra. Because of the great conformational flexibility, these compounds feature a large number of low-energy conformers, and the computed 
conformational ensemble is very dependent on the calculation method, with obvious consequences on the ECD simulations. The situation for $(3 S, 9 S, 11 R)$-dendrodolide A (5) is summarized in Table 2. Very notably, TDDFT calculations run on DFT geometries of $\mathbf{5}$ optimized at B3LYP/6-31G(d) level in vacuo led to a calculated ECD spectrum in almost mirror image relationship with the experimental one. Luckily enough, in the case of dendrodolide A (5) the AC was already known by X-ray crystallography, otherwise a wrong $\mathrm{AC}$ would have been assigned by ECD TDDFT calculations. The result was independent on the functional used in TDDFT calculations (B3LYP, BH\&HLYP and PBE0 were tested), indicating as possible source of error the set of input geometries. In fact, DFT geometry optimizations run at B3LYP/TZVP level and including the PCM solvent model for acetonitrile, led to a different set of conformations (Table 2), though also in this case the TDDFT-calculated Boltzmann-weighted ECD spectrum did not agree with the experimental one. The correct ECD sign could be recovered only by running the geometry optimizations with B97D functional (Table 2). It very interesting to notice that the lowest-energy structures obtained by B3LYP/TZVP and B97D/TZVP were coincident, therefore the difference in the final calculated ECD spectra were due to the higher energy conformers. This finding clearly demonstrates the importance of a correct and complete conformational analysis. Furthermore, for the C9 epimer $(3 S, 9 R, 11 R)$-dendrodolide $\mathrm{B}(\mathbf{6})$, a good agreement between the experimental and calculated ECD spectrum was observed regardless of the method employed in the geometry optimization, i.e., even at B3LYP/6-31G(d) level in vacuo. The case of dendrodolides lends itself as a good proof of several important aspects: a) the impact of even a minor structural difference on the molecular conformation; 2) the strong conformational dependence of ECD; 3) the care which must be put on the generation of input structures during the QM computational procedure.[127]

TABLE 2. Summary of DFT and TDDFT calculation results for $(3 S, 9 S, 11 R)$-dendrodolide A (5).[127]

\begin{tabular}{|c|c|c|}
\hline $\begin{array}{c}\text { Method of } \\
\text { optimization }\end{array}$ & $\begin{array}{c}\text { Number of } \\
\text { conformers }^{\text {(a) }}\end{array}$ & $\begin{array}{c}\text { Agreement of } \\
\text { calculated ECD }^{\text {(b) }}\end{array}$ \\
\hline MMFF & 91 & na \\
\hline B3LYP/6-31G(d) & 10 & NO (mirror image) \\
\hline $\begin{array}{c}\text { B3LYP/TZVP } \\
\text { PCM }^{\text {(c) }}\end{array}$ & 12 & NO (mirror image) \\
\hline $\begin{array}{c}\text { B97D/TZVP, } \\
\text { PCM }^{\text {(c) }}\end{array}$ & 14 & YES \\
\hline
\end{tabular}

(a) For MMFF: energy window of $4.35 \mathrm{kcal} / \mathrm{mol}$; for DFT: population $>1 \%$ at $300 \mathrm{~K}$. (b) TDDFT calculations run with B3LYP, BH\&HLYP and PBE0 functionals and TZVP basis set with PCM solvent model for acetonitrile. The agreement is between the Boltzmann-weighted average calculated ECD spectra and experimental ECD spectra measured in acetonitrile solution. (c) PCM solvent model for acetonitrile.

\subsubsection{The solid-state ECD TDDFT method:}

A very different approach for the determination of the $\mathrm{AC}$ of natural products, including fugal metabolites, is offered by ECD measurements in the solid state. As recalled in $\$ 2.3 .2$ above, many crystalline natural products do not contains the "heavy atoms" able to provide a sufficient anomalous scattering for a reliable AC assignment by the Bijvoet method. In these cases, however, the X-ray structure may still be profitably employed for the AC assignment if used in conjugation with solid-state ECD. In fact, as mentioned in $\$ 3.1$, the ECD spectrum of a crystalline compound may be measured with the so-called pellet technique. This consists in grinding less than $100 \mu \mathrm{g}$ of the crystals with a few mg of spectroscopic-grade $\mathrm{KCl}$ or $\mathrm{KBr}$, and then pressing the mixture with a press (of the same kind employed in IR sample preparation) to obtain a pellet. The ECD spectrum measured on the pellet reflects, in addition to the compound configuration, the exact conformation found in the crystals and measured by X-ray crystallography. The same geometry is therefore employed as input structure in TDDFT calculations (possibly after minimal DFT geometry optimization). In this way, several steps in the computational flowchart (§5.1) are entirely skipped, which not only results in a considerable time-saving, but also avoids many possible sources of errors in the conformational search, geometry optimization, conformer population and so on. The main limitation of this method is of course the availability of single crystals suitable for X-ray analysis. The handling of $\mathrm{KBr}$ or $\mathrm{KCl}$ is perfectly equivalent, but $\mathrm{KCl}$, though less popular, is preferable for its lower cutoff (180 nm vs. 220 $\mathrm{nm}$ for $\mathrm{KBr}$ ). A second drawback is that solid-state ECD measurements require special caution to avoid the presence of artifacts associated with linear dichroism and birefringence.[128] The method is known as the solid-state ECD/TDDFT approach and has been applied to several compounds, mostly secondary metabolites from fungi.[24, 127, 129-140] Other applications are annotated in our dataset (Supplementary Material, Table S1).

An example is viburspiran (9), a polycyclic polyketide containing two maleic anhydride units and an 8-membered ring (Figure 9). Viburspiran was extracted from the endophytic fungus Cryptosporiopsis sp., isolated from the tissues of Viburnum tinus (laurustinus), and exhibited antifungal activity against Botrytis cinerea and Microbotryum violaceum.[132] Viburspiran was obtained as crystalline needles and its X-ray structure determined (Figure 9). However, the anomalous dispersion was too low to measure a meaningful Flack parameter, so the AC could not be established by X-ray crystallography. The solution and solid-state ( $\mathrm{KCl}$ pellet) ECD spectra of viburspiran were consistent and showed a weak positive band at $225 \mathrm{~nm}$ due to the $\mathrm{n}-\pi *$ transitions of the anhydride chromophores, and a negative tail at shorter wavelengths (Figure 10a). Starting from the X-ray geometry, the hydrogen atoms were reoptimized at B3LYP/6-31G(d) level, then TDDFT 
calculations were run with various DFT functionals and the TZVP basis set. In Figure 10b the spectrum computed at the CAM-B3LYP/TZVP level (average for two independent molecules in the crystal cell) is compared with the experimental solid-state ECD spectrum. The established absolute configuration of viburspiran was $(1 R, 2 S, 4 R, 5 R, 6 R, 7 R, 8 S) \cdot[132]$
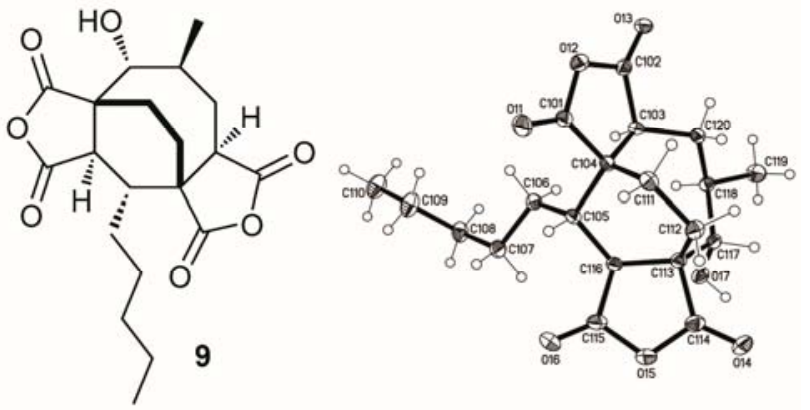

FIGURE 9: Left: structure of $(1 R, 2 S, 4 R, 5 R, 6 R, 7 R, 8 S)$ viburspiran (9). Right: X-ray geometry of one of two independent molecules found per asymmetric unit; the other differed in the conformation of the pentyl group. Reproduced from ref. [132], with permission.
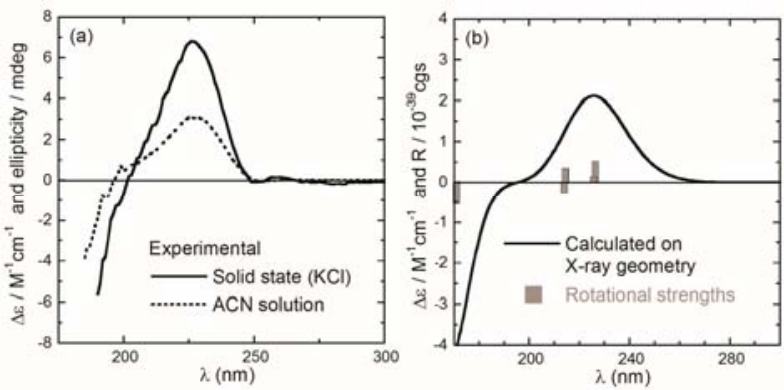

FIGURE 10: (a) Experimental ECD spectra of $(1 R, 2 S, 4 R, 5 R, 6 R, 7 R, 8 S)$-viburspiran (9) measured in the solid state as $\mathrm{KCl}$ pellet and in acetonitrile solution. (b) ECD spectrum calculated at CAM-B3LYP/TZVP level on the Xray geometry (average for two independent molecules in the unit cell). Vertical bars represent calculated rotational strengths. Adapted from ref. [132], with permission.

The use of the above procedure, based on solid-state data rather than on solution-based ones, is especially beneficial in the presence of several chirality centers and/or for very flexible compounds. This is very well demonstrated by the aforementioned case of dendrodolides, for some of which the solid-state TDDFT ECD approach was profitably employed.[127]

\subsection{OR/ORD}

The assignment of the AC of natural products by $a b$ initio OR calculations has been the first computational method applied to metabolites of fungal origin. However, this approach has been soon replaced by more accurate ECD, VCD, and ORD analyses. In a pioneering work Rosini and coworkers faced the problem of the AC assignment of (+)diplopyrone (10, Scheme 6),[141] a phytotoxic monosubstituted tetrahydropyranopyran-2-one isolated as the main phytotoxin from the liquid culture filtrates of Diplodia mutila (Fr.) apud Mont., an endophytic fungus considered one of the main causes of cork oak decline.

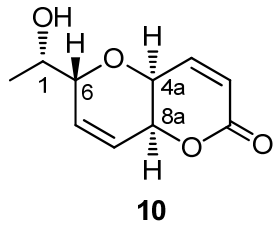<smiles>CC1C=C[C@H]2OC(=O)C=C[C@H]2O1</smiles>

SCHEME 6: Diplopyrone (10) and its truncated model 10a.

The absolute stereochemistry of $\mathrm{C} 1$ was determined to be $(S)$ by the advanced Mosher's method. Extensive NMR analyses allowed the authors to establish a cis junction of the bicyclic system with the two $\mathrm{H} 4 \mathrm{a}$ and $\mathrm{H} 8 \mathrm{a}$ hydrogen atoms opposite to H6.[142] Therefore, (+)-10 could have either $(1 S, 4 \mathrm{a} S, 6 R, 8 \mathrm{a} S)$ or $(1 S, 4 \mathrm{a} R, 6 S, 8 \mathrm{a} R) \mathrm{AC}$. The AC assignment was carried out by analysis of its chiroptical properties, i.e. ECD and OR. The ECD spectrum showed a typical exciton couplet feature deriving from the coupling of the electric dipole allowed transition on the $\alpha, \beta$-unsaturated ester and olefin chromophores. Therefore, a reproduction of the ECD spectrum was attempted by the DeVoe-coupled oscillator (or polarizability) model (mentioned in $\S 4.1$, point 5),[76] arriving at establishing the $(1 S, 4 \mathrm{a} S, 6 R, 8 \mathrm{a} S) \mathrm{AC}$ for (+)-10. Such assignment was confirmed by OR DFT calculations. In order to reduce the computational effort, the conformational analysis was carried out on a simplified model of 10, in which the hydroxylated side chain was substituted by a methyl group (10a in Scheme 6). In fact, the exocyclic side-chain bearing the stereogenic alcohol group does not absorb significantly in the UV-Vis region and, then, does not contribute to the ECD feature. Also its contribution to OR can be considered negligible in comparison to that of the intrinsically chiral and conformationally rigid twisted enone chromophore. Therefore, the $\mathrm{CH}(\mathrm{OH}) \mathrm{CH}_{3}$ moiety can be safely disregarded and replaced by a methyl group. Arbitrarily choosing the $(4 \mathrm{a} S, 6 R, 8 \mathrm{a} S)$ AC, the conformational search carried out at B3LYP/6-31G(d) level of theory in fact provided only two conformers with populations of $78 \%$ and $22 \%$, featuring an equatorial and axial methyl group, respectively (Figure 11).

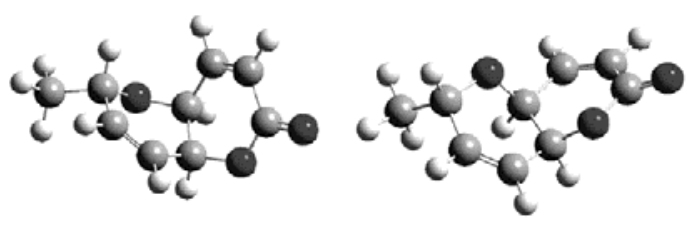

Equatorial conformer (78\%) 
FIGURE 11: Structure of the equatorial and axial conformers (obtained by B3LYP/6-31G(d)) of the $(4 \mathrm{a} S, 6 R, 8 \mathrm{a} S)$-model compound 10a. Adapted with permission from [141]. Copyright 2005 American Chemical Society.

The calculation of the OR at $589 \mathrm{~nm}$ was carried out with the B3LYP functional, using the Sadlej basis set, which is slightly larger than the aug-cc-pVDZ basis set recommended by Stephens et al.[99] The predicted $[\alpha]_{\mathrm{D}}$ value was +35 for the equatorial conformer of $\mathbf{1 0 a}$, and +298 for the axial conformer. The Boltzmann average was +93 , in good agreement with the experimental value of $\mathbf{1 0}$ which spans from +67.6 in $\mathrm{CHCl}_{3}$ to +79 in $\mathrm{MeOH}$. The $(4 \mathrm{a} S, 6 R, 8 \mathrm{a} S) \mathrm{AC}$ was then confirmed for $(+)-\mathbf{1 0}$.

\subsection{VCD}

As discussed above, just a few examples have been reported in the literature in which VCD alone or in conjunction with ECD has been employed for AC assignments of fungal metabolites. Herein we select a couple of those examples which show some interesting features to be discussed.

Some dioxolanone-type secondary metabolites (12-17, Scheme 7) were isolated from the culture filtrates of the fungus Guignardia bidwellii,[143, 144] the causal agent of grape black rot, a devastating disease that threatens vineyards, particularly in Germany. These compounds have in common a core structure similar to that of the previously known metabolite guignardic acid (11) (Scheme 7) and differ only in the substituents at $\mathrm{C} 2$ and the carboxylate function.

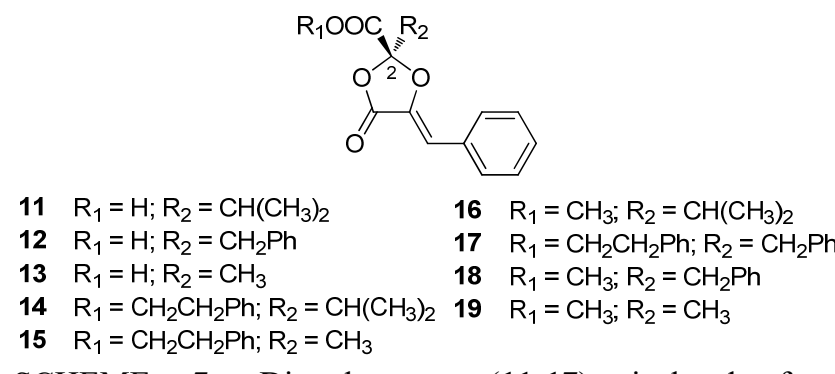

SCHEME 7: Dioxolanones (11-17) isolated from Guignardia bidwellii and methyl ester derivatives (18-19).

The AC of $\mathbf{1 1}$ was already known by total synthesis and comparison of chiroptical data (ECD and OR) with those of the natural product. However, the similar phenguignardic acid (12) showed inverted ECD bands relative to 11. This was clearly due to the presence of an additional phenyl ring which significantly changes the chromophoric system with respect to 11. Therefore, the AC assignment of 12 and 13-17 was carried out by computational simulation of ECD and VCD spectra.[145] Following the standard computational approach described in $\$ 5.1$, a conformational analysis with MMFF force field was first carried out and all obtained conformers subjected to geometry optimization, first at B3LYP/6-31G(d,p) and then at B3LYP/6-311G(d,p) level of theory, taking into account acetonitrile solvation by the IEFPCM model. For the eleven conformers within 2.0 $\mathrm{kcal} / \mathrm{mol}$ with respect to the most stable one, UV and ECD spectra were computed by TD-B3LYP/6-311G(d,p). Unexpectedly, the calculated ECD spectrum of $\mathbf{1 2}$ did not match perfectly the experimental data, therefore the authors resorted to the VCD analysis.[145]

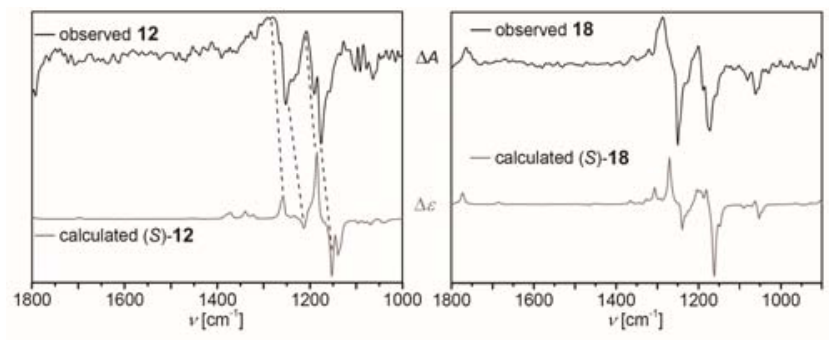

FIGURE 12: Experimental and computed VCD spectra of (S)-phenguignardic acid (12) (left) and its methyl ester (18). Experimental spectra measured in $\mathrm{CDCl}_{3}$ solutions. Calculation level B3LYP/6-311G(d,p), including IEFPCM solvent model. Reproduced from [145] with permission, Copyright Wiley-VCH Verlag GmbH \& Co. KGaA.

Then, the VCD spectrum of $\mathbf{1 2}$ was recorded and compared with the B3LYP/6-311G(d,p)/IEFPCM/ $\mathrm{CHCl}_{3}$ computed one, showing a better, although not fully satisfactory, agreement between theory and experiment. In fact, in the VCD spectrum of $\mathbf{1 2}$ the signs of the major experimental bands were correctly predicted for the $(S)$ enantiomer, while the rotational strengths were not (Figure 12). The authors ascribed the disagreement between the spectra to the known phenomenon of aggregation of carboxylic acids in nonpolar solvents which is notoriously difficult to be taken into account in computations.[146] To remove this difficulty, compound $\mathbf{1 2}$ was transformed into its methyl ester $\mathbf{1 8}$ by treatment with diazomethane. For compound 18 the agreement between the predicted VCD spectrum and the experimental data was much better than for 12 (Figure 12) and, as expected, no significant deviation between calculated and measured IR frequencies was observed. Significantly, the esterification did not improve the consistency of simulated and measured ECD data for 18.[145] The same trends were observed for the pair of alaguignardic acid and its methyl ester (13 and 19, Scheme 7) in which the agreement of the VCD spectra was again improved in the ester. VCD analysis was also applied to naturally occurring esters 14-17, which showed a very similar VCD pattern predicted with high accuracy by calculations for the $(S)$ configuration.[145] In these cases VCD predictions showed to provide more accurate results than ECD. Moreover, it was demonstrated that simple chemical modifications, like esterifications, can help in simplifying the computational treatment removing aggregation effects which are difficult to reproduce computationally. 
Another example in which VCD calculations were successfully employed to assign the AC of fungal phytotoxins is provided by oxalicumone C (20) (Scheme 8), isolated in the marine-derived fungus Penicillium oxalicum.[147] Such phytotoxin belongs to the family of chromones, naturally occurring compounds often isolated from different fungal species. Some of them also present interesting activity as plant growth regulator, while others display moderate cytotoxicity against cancer cell lines. The total synthesis of racemic 20, its enantiomeric separation by chiral HPLC, and the elucidation its AC by computational analysis of ECD and VCD spectra has been reported by Opatz, Waldvogel and coworkers.[148]

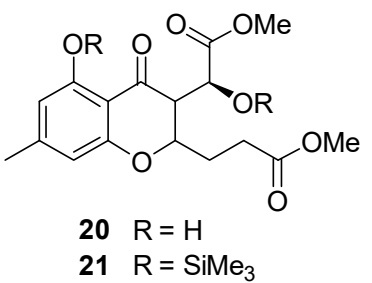

SCHEME 8: Oxalicumone C (20) and silyl ether (21).

For the determination of the AC of compound $\mathbf{2 0}$ the standard computational approach described in $\$ 5.1$ was again applied. Therefore, MMFF conformational analysis was carried out followed by geometry optimization, first at B3LYP/6-31G(d,p) and then at B3LYP/6$311 \mathrm{G}(\mathrm{d}, \mathrm{p}) / \mathrm{IEFPCM} /$ acetonitrile level of theory, which also provided the Boltzmann-averaged VCD spectrum. The UV and ECD spectra were instead obtained at TD-B3LYP/6$311 \mathrm{G}(\mathrm{d}, \mathrm{p}) / \mathrm{IEFPCM}$ level.[148] The calculated ECD spectrum of $\mathbf{2 0}$ showed good agreement with the experimental data, while the VCD spectrum of $\mathbf{2 0}$ did not match well with the calculated one. The authors attributed this disagreement to the presence of intermolecular hydrogen bonding of the two hydroxy groups, which is difficult to represent in silico. Therefore, both hydroxy groups of compound 20 were silylated to obtain the bis(trimethylsilyl) ether 21. Calculation of the vibrational spectra of compound 21 was performed in the same way as described for $\mathbf{2 0}$ starting with 303 candidate conformers and ending up with 38 conformers within a $2 \mathrm{kcal} / \mathrm{mol}$ window. In the absence of the hydrogen bonds, the measured and the calculated VCD spectra of 21 showed good agreement over the measured spectral range $\left(1050-1800 \mathrm{~cm}^{-1}\right)$. Therefore the $(S)$ configuration was attributed to the first eluting enantiomer of 20 and the $(R)$ configuration to the second eluting enantiomer.[148] Finally, the AC of the natural product was determined to be $(S)$ by comparing its OR $\left([\alpha]_{\mathrm{D}} 20=+11.25\right.$ $\left(c=0.12, \mathrm{CHCl}_{3}\right)$ ) with those of the two synthesized enantiomers $(S)-(+)-20$ and $(R)-(-)-20 .[147]$
Although the independent application of ORD, ECD, and VCD calculations has proved to be practical and reliable for AC assignments, in some cases, especially when the molecule displays high conformational flexibility, some uncertainties in the assignment are unavoidable. Therefore, for flexible molecules including some natural products, the concerted application of more than one chiroptical method has emerged as a viable approach.[63, 125, 149, 150] An interesting application of this approach concerns the AC assignment of fungal phytotoxins phyllostin (22) and scytolide (23), two hexahydro-1,4-benzodioxines produced by Phyllosticta cirsii, and oxysporone (24), a dihydrofuropyranone recently isolated from a strain of Diplodia Africana (Scheme 9).[151]

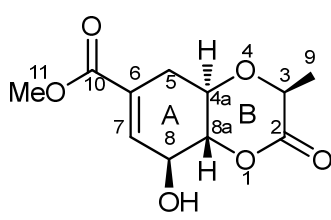

$(-)-(3 S, 4 a R, 8 S, 8 a R)-22$

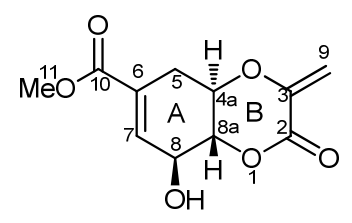

$(-)-(4 a R, 8 S, 8 a R)-23$

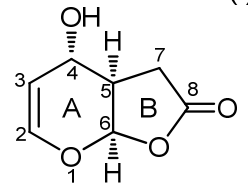

$(+)-(4 S, 5 R, 6 R)-24$

SCHEME 9: Structures and assigned AC of phyllostin (22), scytolide (23), and oxysporone (24).

The RC of 22-24 was known by NMR[152-154] and Xray analyses.[155] Therefore, following the standard computational approach described in $\$ 5.1$, a preliminary MM conformational search was first carried out on the chosen stereoisomers $(3 S, 4 \mathrm{a} R, 8 S, 8 \mathrm{a} R)-\mathbf{2 2},(4 \mathrm{a} R, 8 S, 8 \mathrm{a} R)-\mathbf{2 3}$, and $(4 R, 5 S, 6 S)-24$. After surveying the conformational space, the geometries within a $10 \mathrm{kcal} / \mathrm{mol}$ energy window were subjected to energy minimization at B3LYP/TZVP level for $\mathbf{2 2}$ and $\mathbf{2 3}$, while for $\mathbf{2 4}$ different combinations of basis sets as well as gas phase/solvent were employed. After optimization at B3LYP/TZVP level in gas phase, four stable conformers were obtained for both $\mathbf{2 2}$ and $\mathbf{2 3}$, while B3LYP/cc-pVTZ optimizations of $\mathbf{2 4}$ provided six conformers. In Figure 13 the conformers accounting for $97 \%$ of the overall population for each compound are reported.

\subsection{ECD, VCD and ORD}



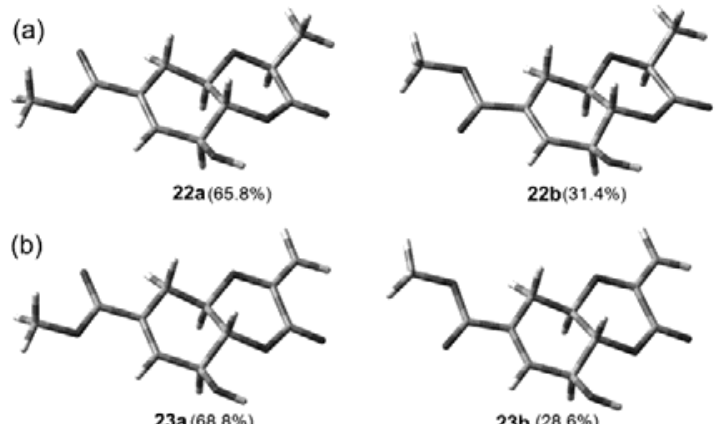

(c)
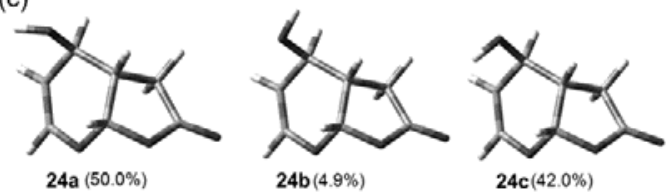

FIGURE 13: Most stable conformers of $(3 S, 4 \mathrm{a} R, 8 S, 8 \mathrm{a} R)-\mathbf{2 2}$ (a), $(4 \mathrm{a} R, 8 S, 8 \mathrm{a} R)-\mathbf{2 3}$ (b), and (4R,5S,6S)-24 (c) optimized by B3LYP/cc-pVTZ. Relative populations are in parentheses. Adapted with permission from ref. [151]. Copyright 2013 American Chemical Society.

At first, an attempt to assign the AC by comparison of experimental and computed ORD curves was carried out. The experimental curves were measured for $(-)-\mathbf{2 2},(-)-\mathbf{2 3}$, and $(+)-24$ at four wavelengths $(589,546,435$ and $405 \mathrm{~nm})$, where they appear as plain positive or negative curves (Figures 14-16). Theoretical ORD curves were obtained by calculations at TD-B3LYP/aug-cc-pVDZ level on the major conformers, followed by Boltzmann averaging.
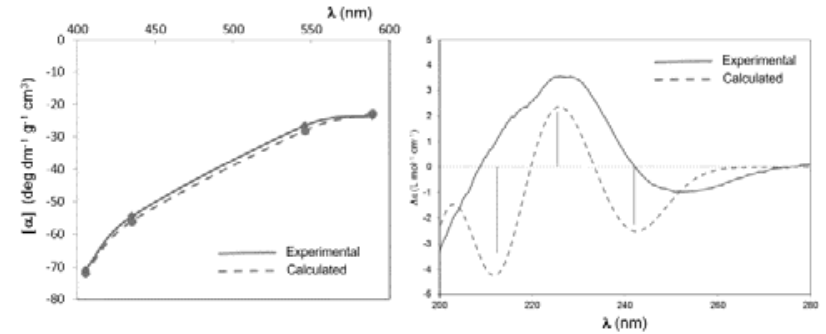

FIGURE 14: Experimental and computed ORD curve (left) and ECD spectra (right) of $(3 S, 4 \mathrm{a} R, 8 S, 8 \mathrm{a} R)-22$. Vertical bars in the right panel represent calculated rotational strengths. Experimental spectra measured in methanol. Calculation level: OR, B3LYP/aug-cc-pVDZ; ECD, CAMB3LYP/aug-cc-pVDZ. Adapted with permission from ref. [151]. Copyright 2013 American Chemical Society.

Comparisons with the experimental data shows that for 22 the trend and sign of the experimental ORD is well reproduced by the theoretical curve (Figure 14), supporting the assignment of the $(3 S, 4 \mathrm{a} R, 8 S, 8 \mathrm{a} R)$ AC for (-)-22. For 24 the calculated ORD is a plain negative curve appearing as the mirror image of the experimental ones (Figure 15) and thus it is allied to enantiomeric structures. Then for $\mathbf{2 4}$ the $(4 S, 5 R, 6 R) \mathrm{AC}$, opposite to the one chosen for calculations, has to be assigned. Computational ORD analysis of compound 23 instead failed to provide a reliable AC assignment (Figure 16), even using different functionals and basis sets, as well as solvation models in calculations. This result highlights the necessity to resort to more than a single approach to obtain reliable $\mathrm{AC}$ assignments.

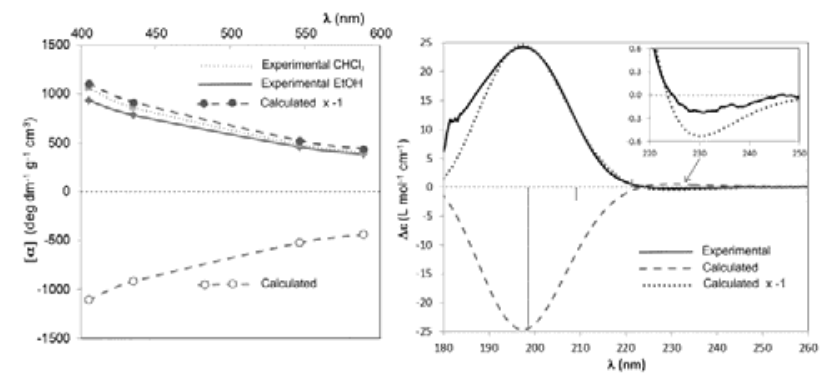

FIGURE 15: Experimental and computed ORD curve (left) and ECD spectra (right) of 24. Vertical bars in the right panel represent calculated rotational strengths. Experimental spectra measured in methanol (and chloroform for ORD). Calculation level: OR, B3LYP/aug-cc-pVDZ; ECD, CAMB3LYP/aug-cc-pVDZ. Adapted with permission from ref. [151]. Copyright 2013 American Chemical Society.

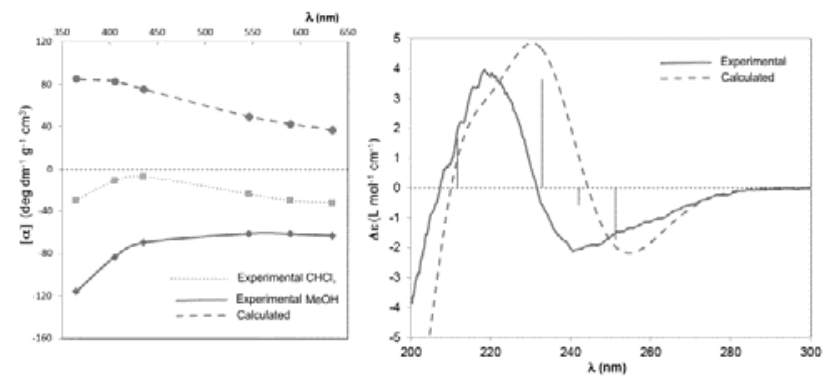

FIGURE 16: Experimental and computed ORD curve (left) and ECD spectra (right) of $(4 S, 5 R, 6 R)-23$. Vertical bars in the right panel represent calculated rotational strengths. Experimental spectra measured in methanol (and chloroform for ORD). Calculation level: OR, B3LYP/aug-cc-pVDZ; ECD, CAM-B3LYP/aug-cc-pVDZ. Adapted with permission from ref. [151]. Copyright 2013 American Chemical Society.

Therefore, also ECD and VCD spectral analyses were carried out. ECD spectra of (-)-22, (-)-23, and (+)-24 were recorded in the 200-300 $\mathrm{nm}$ range and ECD calculations were performed at the TD-CAM-B3LYP/aug-cc-pVDZ level of theory on the most populated conformers. Comparison between the calculated and experimental ECD spectra of (-)22 shows a satisfactory, although not perfect, match (Figure 14). The calculation in fact reproduced the sign and intensity of the main experimental CEs at 250 and $225 \mathrm{~nm}$, supporting the assignment of $(3 S, 4 \mathrm{a} R, 8 S, 8 \mathrm{a} R)$ AC to natural compound $(-)-22$, in agreement with ORD analysis. In 23 a good agreement was found between the experimental and simulated ECD spectra (Figure 16), thus providing, on the contrary of the ORD analysis, an unambiguous evidence for the AC assignment for natural (-)-23 as $(4 \mathrm{a} R, 8 S, 8 \mathrm{a} R)$. Finally, the computed and experimental ECD spectra for $(4 R, 5 S, 6 S)-24$ and $(+)-24$ were almost superimposable, but in 
a near mirror image relationship, strongly supporting the opposite $(4 S, 5 R, 6 R)$ AC for (+)-24 (Figure 15). The final proof of the AC assignment was attempted by VCD analysis.
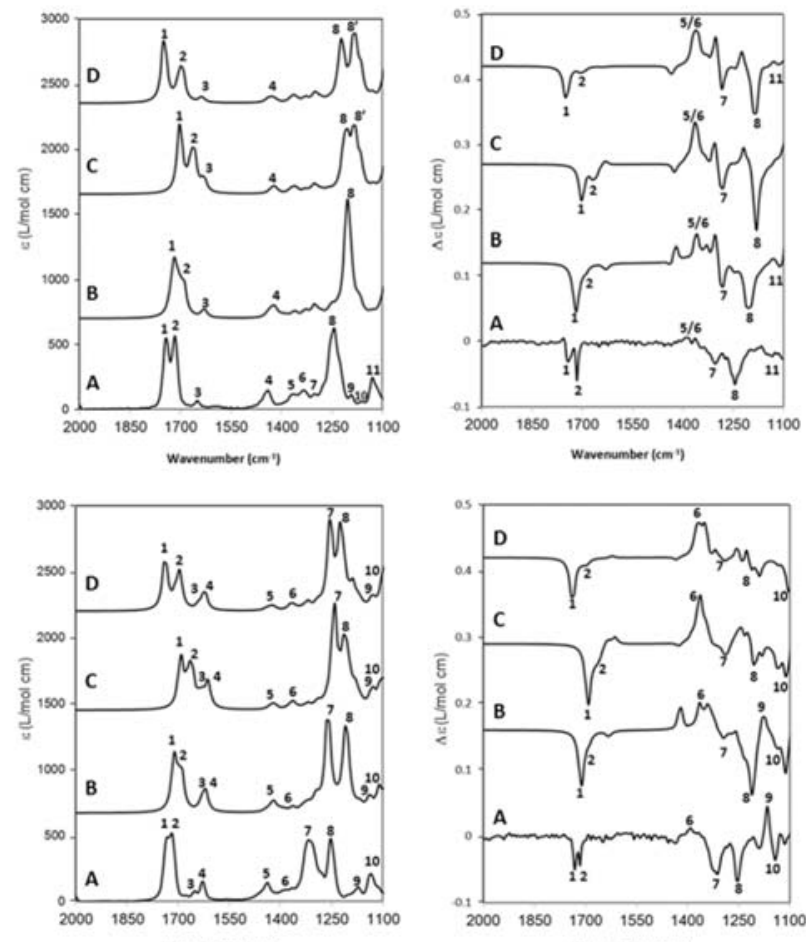

FIGURE 17: IR (left) and VCD (right) spectra of 22 (top panel) and 23 (bottom panel). Trace A: experimental (DMSO-d 6 solutions); B: B3LYP/TZVP with explicit solvent (DMSO); C: B3LYP/TZVP with implicit solvent (IEFPCM, DMSO); D: B3LYP/TZVP, gas-phase. The numbers on VCD and IR signals refer to the normal modes. Adapted with permission from ref. [151]. Copyright 2013 American Chemical Society.

The IR and VCD spectra of (-)-22, (-)-23, and (+)-24 were recorded in the mid-IR region $\left(800-2000 \mathrm{~cm}^{-1}\right)$ and compared with the calculated ones (Figure 17). To improve the correlation between the experimental and calculated spectra, both implicit DMSO (IEFPCM) [108] and explicit[91] solvent models were applied, observing a redistribution of the Boltzmann populations of the various minima with respect to the gas phase. The explicit solvation model, taking into account specific intermolecular solutesolvent interactions, was obtained by positioning a DMSO molecule interacting with a hydroxy group for the various conformers, and these assemblies were optimized at the B3LYP/TZVP level of theory. Calculated IR and VCD spectra for 22 in the gas phase, and with the implicit (IEFPCM) and explicit DMSO solvation model are compared with the experimental spectra in Figure 17. The signs of calculated and experimental VCD bands are in good agreement, the best match being observed with the explicit model (trace B), thus increasing the confidence level for the assignment of $(3 S, 4 \mathrm{a} R, 8 S, 8 \mathrm{a} R) \mathrm{AC}$ to (-)-22. For (-)-23 the spectral envelope in the region below $1500 \mathrm{~cm}^{-1}$ provides additional evidence for the presence of multiple stable conformers. The comparison between experimental and calculated IR and VCD spectra show a satisfactory qualitative experimental-calculated correlation, supporting the (-)- $(4 \mathrm{a} R, 8 S, 8 \mathrm{a} R)-\mathbf{2 3}$ assignment. The experimentalcalculated spectra comparison (not shown here) indicates that the experimental VCD bands of $(+)-24$ do not match the simulated data for the chosen model $(4 R, 5 S, 6 S)-24$ but, on the contrary, they match the calculated VCD trace for the enantiomer $(4 S, 5 R, 6 R)-\mathbf{2 4}$, although not in an optimal way.[151] Resorting to a larger level basis sets did not resolve these inconsistencies, therefore a quantitative assessment of the reliability of calculated VCD bands was also carried out by the so-called robustness or $\zeta$-factor analysis.[156,157] In fact, for flexible compounds displaying a pool of conformers with similar Boltzmann populations and multiple transitions beneath VCD bands, the overall experimental VCD spectrum often differs from those calculated for the individual conformers, thus making difficult to establish a one-to-one band correspondence. In these situations, it is beneficial to switch from the usual visual comparison to a quantitative comparison of VCD and IR bands, as well as of their ratio.[116,117] The $g$-factor (eq. 4 above) is related to the $\zeta$-factor, the ratio of rotational and dipole strengths $(\mathrm{R} / \mathrm{D})$, by $g=4 \zeta$. Moreover, the $\zeta$-factor lends itself to judging the robustness of a calculated VCD/IR band; a criterion $\zeta>10 \mathrm{ppm}$ has been suggested.[157] The $\zeta$-factor was calculated for the main VCD transitions of compounds 22-24. For $(3 S, 4 \mathrm{a} R, 8 S, 8 \mathrm{a} R)-\mathbf{2 2}$ three VCD bands involving the vibrational motion of each of the four stereogenic centers, for $(4 \mathrm{a} R, 8 S, 8 \mathrm{a} R)-\mathbf{2 3}$ four out of seven bands, and for $(4 S, 5 R, 6 R)-24$ nine bands, displayed satisfactory $\zeta$-factor values providing a higher quantitative level of confidence for the VCD-based AC assignment. Then, in these cases only the concerted application of the three chiroptical spectroscopies resolved any ambiguity in the AC assignment. In fact, in the case of $\mathbf{2 2}$, the theoretical ORD curve fully matched the experimental data, while ECD and VCD predictions showed some differences with respect to the experiment. For 23, the opposite was true, and only ECD and VCD allowed a reliable AC assignment. Finally, the good agreement between experimental and calculated ORD, ECD, and VCD spectra of $\mathbf{2 4}$, led to an unequivocal AC assignment.

A final interesting example is provided by the $\mathrm{AC}$ assignment of the fungal phytotoxin seiricardine A (25) (Scheme 10), which has been established by a combined analysis of ORD, ECD, and VCD properties of a suitable chromophoric derivative.[158] Compound 25 was produced by some Seiridium fungi associated with the canker disease of Italian cypress and displays several promising bioactive properties as antimalarial, anticancer, and possible natural fungicide against other fungi pathogenic of cypress plant. 


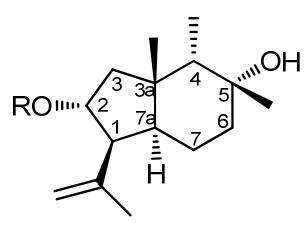

$25 \mathrm{R}=\mathrm{H}$

$26 \mathrm{R}=4-\mathrm{BrPhCO}$

SCHEME 10: Structure of $(1 S, 2 R, 3 \mathrm{a} S, 4 S, 5 R, 7 \mathrm{a} S)$ seiricardine A (25), and its $p$-Br-benzoyl ester (26).

The AC assignment of (-)-25 is a quite challenging task because this bicyclic sesquiterpene, lacking chromophores absorbing in the UV-Vis region, presents a quite weak UV spectrum, a nearly undetectable ECD spectrum, low OR values, and even a weak VCD spectrum. Therefore, in order to make possible the determination of $\mathrm{AC}$ of (-)-25 by chiroptical methods, a strong chromophore was introduced transforming 25 into the corresponding 2-O- $p$ bromobenzoate ester (-)-26 (Scheme 10) possessing favorable properties for ECD/ORD. Taking into account the known $\left(1 R^{*}, 2 S^{*}, 3 \mathrm{a} R^{*}, 4 R^{*}, 5 R^{*}, 7 \mathrm{a} R^{*}\right) \mathrm{RC}$ of $25,[159]$ a conformational analysis was carried out in gas phase at B3LYP/TZVP level of theory on the enantiomer $(1 R, 2 S, 3 \mathrm{a} R, 4 R, 5 R, 7 \mathrm{a} R)-\mathbf{2 6}$, providing seven appreciably populated conformers (the two most stable are shown in Figure 18).
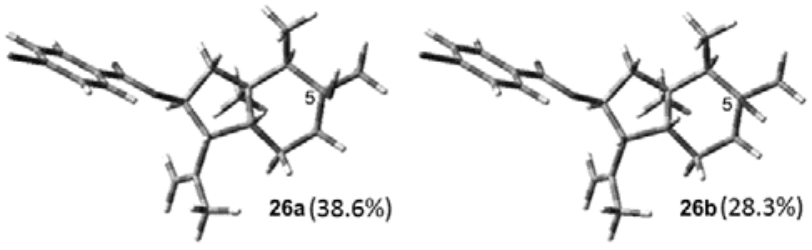

FIGURE 18: Most stable conformers of $\mathbf{2 6}$ optimized by B3LYP/TZVP. Relative populations in parentheses. Adapted from [158]. Copyright 2015, with permission from Elsevier.

The ECD spectrum recorded in methanol in the 200-350 $\mathrm{nm}$ range exhibits a negative Cotton effect (CE) at $251 \mathrm{~nm}$, and two other negative CEs at $218 \mathrm{~nm}$ and $203 \mathrm{~nm}$ (Figure 19). The UV and ECD spectra of $(1 R, 2 S, 3 \mathrm{a} R, 4 R, 5 S, 7 \mathrm{a} R)-\mathbf{2 6}$ were calculated at the TD-CAM-B3LYP/6-311+G(d,p)/ IEFPCM/methanol level on the previously found conformers and Boltzmann averaged over the conformers populations. The comparison of the experimental ECD spectrum for (-)26 and the computed one for $(1 R, 2 S, 3 \mathrm{a} R, 4 R, 5 S, 7 \mathrm{a} R)-\mathbf{2 6}$ shows that this latter reproduces the overall ECD profile, but in a mirror image fashion (Figure 19). The good spectral reproduction removes any uncertainty in the comparison, leading to a reliable assignment of $(1 S, 2 R, 3 \mathrm{a} S, 4 S, 5 R, 7 \mathrm{a} S)$ AC for (-)-26 and also for the natural (-)-25. The introduction of the $p$-bromobenzoate moiety provided a twofold benefit, both improving the electronic properties of 26 and reducing the molecular conformational mobility.

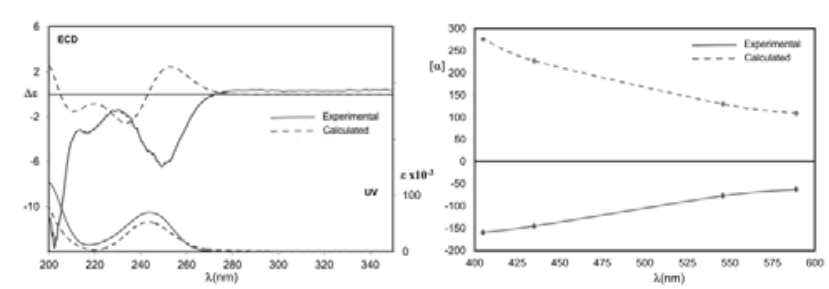

FIGURE 19: Experimental and computed ECD spectra (left) and ORD curve (right) of 26. Experimental spectra measured in methanol. Calculation level: ORD, B3LYP/6$311+\mathrm{G}(\mathrm{d}, \mathrm{p}) ; \quad \mathrm{ECD}, \quad$ CAM-B3LYP/6-311+G(d,p), both including IEFPCM for methanol. Adapted from [158]. Copyright 2015, with permission from Elsevier.

A further confirmation of the configurational assignment was obtained by a computational analysis of the ORD curve. The ORD data of (-)-26 were recorded in methanol obtaining a plain negative curve (Figure 19). ORD data for $(1 R, 2 S, 3 \mathrm{a} R, 4 R, 5 S, 7 \mathrm{a} R)-26$ were then calculated on the previously found conformers at TD-B3LYP/6-311+G(d,p) level in the same solvent by IEFPCM model and Boltzmann averaged over the conformers populations. The comparison with experimental data shows (Figure 19) that the computed ones are opposite in sign giving rise to a positive plain curve, thus supporting the previous assignment.

The derivatization of $\mathbf{2 5}$ into $\mathbf{2 6}$ was not only needed to obtain a more useful ECD spectrum, but also a more sensitive VCD response. Preliminary measurements of underivatized 25 revealed a lack of any diagnostic feature in the VCD spectrum and a very low signal/noise ratio. In the case of $\mathbf{2 6}$ the use of VCD for stereochemical assignments was quite challenging because both IR and VCD responses varied among the conformers and the most highly populated conformer was only 38\% (Figure 18). Taking into account that the experimental measurements were done in methanol, a solvent capable of H-bonding with the oxygenated hydroxyl ester 26, a theoretical model with an explicit solvent model was considered. To this end two molecules of $\mathrm{CH}_{3} \mathrm{OH}$ set at $2.1 \AA$ away from 26 were added and the disposition of the explicit solvents explored via a MM/MC search. Subsequent DFT geometry optimizations at B3LYP/TZVP level of theory provided six stable conformers. IR and VCD spectra of $(1 R, 2 S, 3 \mathrm{a} R, 4 R, 5 S, 7 \mathrm{a} R)$ 26 were then computed and Boltzmann averaged. As revealed by Figure 20, a good correlation between the experimental and theoretical data was obtained for all twelve IR bands, while the correlation of only four VCD bands was observed. However, the mirror-image relationship of these four diagnostic bands indicates that the AC assignment of ()-26 in solution should be opposite of that $(1 R, 2 S, 3 \mathrm{a} R, 4 R, 5 S, 7 \mathrm{a} R)-\mathbf{2 6}$ chosen for calculations, in keeping again with the results from the other spectroscopies. It has to be noted that in case of $\mathbf{2 6}$ the AC assignment cannot be made independently by the VCD method alone. The experimental signals were fairly weak while a number of 
theoretical VCD bands remained uncorrelated, regardless of the level of theory used.

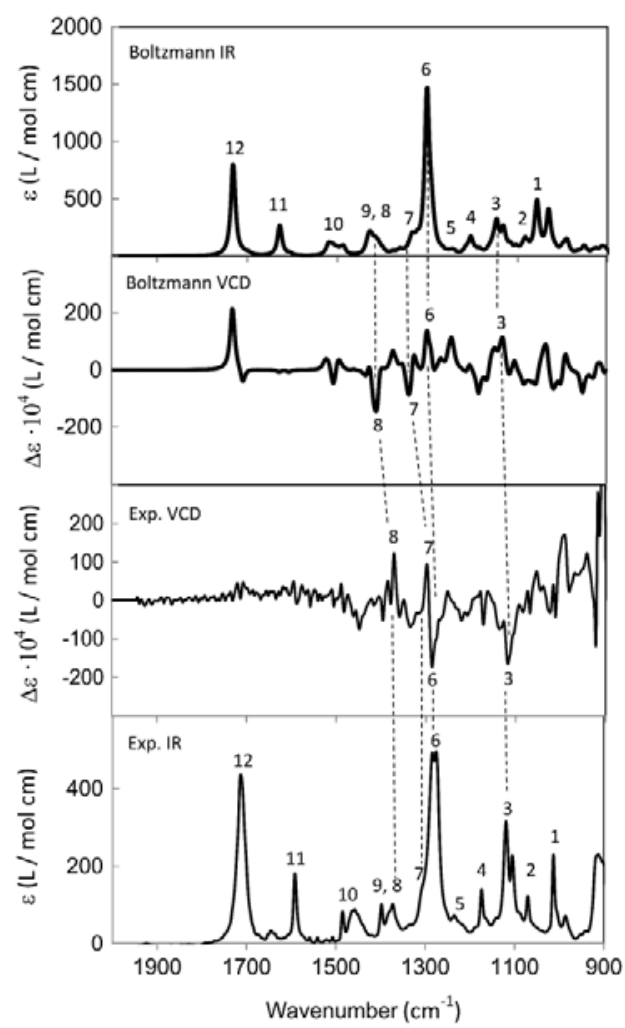

FIGURE 20: Experimental $\left(\mathrm{CD}_{3} \mathrm{OD}\right)$ and computed IR and VCD spectra of 26. Calculation level B3LYP/TZVP. The numbers on VCD and IR signals refer to the normal modes. Adapted from [158]. Copyright 2015, with permission from Elsevier.

\section{CONCLUSIONS}

The quantum mechanical calculation of chiroptical properties has emerged in the last decade as a versatile and reliable approach for the $\mathrm{AC}$ assignment of natural products, including many fungal metabolites. The main advantage of such approach over the diffractometric method lies on the possibility to achieve chiroptical measurements in solution and, often, on a microscale, thus allowing one to treat also non-crystalline products. Moreover, such approach is essentially non-empirical and not limited by the presence on the molecule of specific functional groups, thus being superior in versatility with respect to the NMR methods based on the derivatization of functional groups such as alcohols, amine, and carboxylic acids. As shown in the present review, ECD, ORD, and VCD are often complementary and, when necessary, their concerted application can help one to overcome ambiguities arising in the AC assignment. Nowadays small molecules up to 200-300 atoms can be treated without great computational effort, thus allowing this type of computations to be run also on desktop computers. This fact, joined to the availability of both commercial and freeware computational packages, has led to a great diffusion in the application of these methods. It must be however kept in mind that to have reliable and consistent results these methods cannot be applied in a black box manner and that a careful choice of the most appropriate computational protocol and of the suitable experimental condition of measurement is always compulsory.

\section{ACKNOWLEDGEMENTS}

S. S. and P. S. acknowledge University of Basilicata for financial support (R.I.L. 2015 grant). M. G. acknowledges the support from the Polish Ministry of Science and Higher Education ('Mobilnosc Plus' grant 1286/MOB/IV/2015/0).

\section{REFERENCES}

[1] Lin, G.-Q.; You, Q.-D.; Cheng, J.-F., Eds. Chiral Drugs: chemistry and biological action Wiley: Hoboken, NJ, 2011.

[2] Nunez, M.C.; Garcia-Rubino, M.E.; Conejo-Garcia, A.; Cruz-Lopez, O.; Kimatrai, M.; Gallo, M.A.; Espinosa, A.; Campos, J.M., Homochiral Drugs: A Demanding Tendency of the Pharmaceutical Industry. Curr. Med. Chem., 2009, 16(16), 2064-2074.

[3] Branch, S.K.; Hutt, A.J. In Drug Stereochemistry; CRC Press, 2012, pp 240-273.

[4] Brooks, W.H.; Guida, W.C.; Daniel, K.G., The Significance of Chirality in Drug Design and Development. Curr. Top. Med. Chem., 2011, 11(7), 760-770.

[5] Miller, S. Scientific and Regulatory Aspects of Quality Control for Chiral Drugs. In 18th International Symposium on Chirality: Busan, 2006.

[6] Sekhon, B.S., Exploiting the Power of Stereochemistry in Drugs: An Overview of Racemic and Enantiopure Drugs. $J$. Mod. Med. Chem., 2013, 1, 10-36.

[7] Challener, C., Expanding the Chiral Toolbox. Pharm. Technol., 2016, 40(7), 28-29.

[8] Newman, D.J.; Cragg, G.M. In Comprehensive Natural Products II. Mander, L.; Liu, H.-W., Eds.; Elsevier: Oxford, 2010; Vol. 2, pp 623-650.

[9] Butler, M.S.; Robertson, A.A.B.; Cooper, M.A., Natural product and natural product derived drugs in clinical trials. Nat. Prod. Rep., 2014, 31(11), 1612-1661.

[10] Newman, D.J.; Cragg, G.M., Natural Products as Sources of New Drugs from 1981 to 2014. J. Nat. Prod., 2016, 79(3), 629-661.

[11] Cragg, G.M.; Newman, D.J.; Kingston, D.G.I. In Comprehensive Natural Products II. Mander, L.; Liu, H.-W., Eds.; Elsevier: Oxford, 2010; Vol. 2, pp 5-39.

[12] Gunatilaka, A.A.L., Natural Products from PlantAssociated Microorganisms: Distribution, Structural Diversity, Bioactivity, and Implications of Their Occurrence. J. Nat. Prod., 2006, 69(3), 509-526.

[13] Kusari, S.; Lamshöft, M.; Kusari, P.; Gottfried, S.; Zühlke, S.; Louven, K.; Hentschel, U.; Kayser, O.; Spiteller, 
M., Endophytes Are Hidden Producers of Maytansine in Putterlickia Roots. J. Nat. Prod., 2014, 77(12), 2577-2584.

[14] Krastel, P.; Petersen, F.; Roggo, S.; Schmitt, E.; Schuffenhauer, A. In Chirality in Drug Research. Francotte, E.; Lindner, W., Eds.; Wiley-VCH: Weinheim, 2006, pp 6794.

[15] Finefield, J.M.; Sherman, D.H.; Kreitman, M.; Williams, R.M., Enantiomeric Natural Products: Occurrence and Biogenesis. Angew. Chem. Int. Ed., 2012, 51(20), 48024836.

[16] Mori, K., Bioactive natural products and chirality. Chirality, 2011, 23(6), 449-462.

[17] Harada, N.; Nakanishi, K. Circular Dichroic Spectroscopy - Exciton Coupling in Organic Stereochemistry. University Science Books: Mill Valley, CA, 1983.

[18] Bijvoet, J.M.; Peerdeman, A.F.; van Bommel, A.J., Determination of the Absolute Configuration of Optically Active Compounds by Means of X-Rays. Nature, 1951, 168(4268), 271-272.

[19] Eliel, E.L.; Wilen, S.H. Stereochemistry of organic compounds. Wiley: New York, 1994.

[20] Berova, N.; Polavarapu, P.L.; Nakanishi, K.; Woody, R.W., Eds. Comprehensive Chiroptical Spectroscopy. John Wiley \& Sons, Inc.: Hoboken, NJ, 2012.

[21] Polavarapu, P.L. Chiroptical Spectroscopy: Fundamentals and Applications. CRC Press: Boca Raton, FL, 2016.

[22] Polavarapu, P.L., Renaissance in chiroptical spectroscopic methods for molecular structure determination. Chem. Rec., 2007, 7(2), 125-136.

[23] Bringmann, G.; Bruhn, T.; Maksimenka, K.; Hemberger, Y., The Assignment of Absolute Stereostructures through Quantum Chemical Circular Dichroism Calculations. Eur. J. Org. Chem., 2009, 2009(17), 2717-2727.

[24] Pescitelli, G.; Kurtán, T.; Flörke, U.; Krohn, K., Absolute structural elucidation of natural products-A focus on quantum-mechanical calculations of solid-state $\mathrm{CD}$ spectra. Chirality, 2009, 21(1E), E181-E201.

[25] Li, X.C.; Ferreira, D.; Ding, Y., Determination of Absolute Configuration of Natural Products: Theoretical Calculation of Electronic Circular Dichroism as a Tool. Curr. Org. Chem., 2010, 14(16), 1678-1697.

[26] He, Y.; Wang, B.; Dukor, R.K.; Nafie, L.A., Determination of absolute configuration of chiral molecules using vibrational optical activity: a review. Appl. Spectr., 2011, 65(7), 699-723.

[27] Sheng-Ding, Z.; Lan, S.; Du-Qiang, L.; Hua-Jie, Z., Progression of Absolute Configuration Determination in Natural Product Chemistry Using Optical Rotation (Dispersion), Matrix Determinant and Electronic Circular Dichroism Methods. Curr. Org. Chem., 2011, 15(11), 18431862.
[28] Bertucci, C.; Tedesco, D., Advantages of electronic circular dichroism detection for the stereochemical analysis and characterization of drugs and natural products by liquid chromatography. J. Chromatogr. A, 2012, 1269, 69-81.

[29] Molinski, T.F.; Morinaka, B.I., Integrated Approaches to the Configurational Assignment of Marine Natural Products. Tetrahedron, 2012, 68(46), 9307-9343.

[30] Kong, L.-Y.; Wang, P., Determination of the absolute configuration of natural products. Chin. J. Nat. Med., 2013, 11(3), 193-198.

[31] Batista, J.M.; da Silva Bolzani, V. In Stud. Nat. Prod. Chem. Rahman, A.u., Ed.; Elsevier: Amsterdam, 2014; Vol. 41, pp 383-417.

[32] Nugroho, A.E.; Morita, H., Circular dichroism calculation for natural products. J. Nat. Med., 2014, $68(1), 1$ 10.

[33] Batista Jr, J.M.; Blanch, E.W.; Bolzani, V.d.S., Recent advances in the use of vibrational chiroptical spectroscopic methods for stereochemical characterization of natural products. Nat. Prod. Rep., 2015, 32(9), 1280-1302.

[34] Joseph-Nathan, P.; Gordillo-Román, B. In Progress in the Chemistry of Organic Natural Products. Kinghorn, A.D.; Falk, H.; Kobayashi, J., Eds.; Springer: Cham, Switzerland, 2015; Vol. 100, pp 311-452.

[35] Polavarapu, P.L.; Petrovic, A.G.; Vick, S.E.; Wulff, W.D.; Ren, H.; Ding, Z.; Staples, R.J., Absolute Configuration of 3,3'-Diphenyl-[2,2'-binaphthalene]-1,1'-diol Revisited. J. Org. Chem., 2009, 74(15), 5451-5457.

[36] Silverstein, R.M.; Webster, F.X.; Kiemle, D.J.; Bryce, D.L. Spectrometric Identification of Organic Compounds. 8th ed. Wiley: New York, 2015.

[37] Edison, A.S.; Schroeder, F.C. In Comprehensive Natural Products II. Mander, L.; Liu, H.-W., Eds.; Elsevier: Oxford, 2010; Vol. 9, pp 169-196.

[38] Elyashberg, M.E.; Williams, A.J.; Martin, G.E., Computer-assisted structure verification and elucidation tools in NMR-based structure elucidation. Prog. Nucl. Magn. Reson. Spectrosc., 2008, 53(1-2), 1-104.

[39] Amagata, T. In Comprehensive Natural Products II. Mander, L.; Liu, H.-W., Eds.; Elsevier: Oxford, 2010; Vol. 2, pp 581-621.

[40] Nicolaou, K.C.; Snyder, S.A., Chasing Molecules That Were Never There: Misassigned Natural Products and the Role of Chemical Synthesis in Modern Structure Elucidation. Angew. Chem. Int. Ed., 2005, 44(7), 1012-1044.

[41] Jegorov, A.; Hušák, M. In Natural Products Analysis. Havlíček, V.; Spížek, J., Eds.; John Wiley \& Sons, Inc, 2014, pp 285-311.

[42] Bifulco, G.; Dambruoso, P.; Gomez-Paloma, L.; Riccio, R., Determination of Relative Configuration in Organic Compounds by NMR Spectroscopy and Computational Methods. Chem. Rev., 2007, 107(9), 37443779 . 
[43] Contreras, R.H.; Peralta, J.E., Angular dependence of spin-spin coupling constants. Prog. Nucl. Magn. Reson. Spectrosc., 2000, 37, 321-425.

[44] Mori, K. In Comprehensive Natural Products II. Mander, L.; Liu, H.-W., Eds.; Elsevier: Oxford, 2010; Vol. 9, pp 147-167.

[45] Polavarapu, P.L., Structural Analysis Using Chiroptical Spectroscopy: Insights and Cautions. Chirality, 2016, 28(6), 445-452.

[46] Dictionary of Natural Products, v. 25.1, Taylor \& Francis: 2016.

[47] Young, B.L.; Cooks, R.G., Improvements in quantitative chiral determinations using the mass spectrometric kinetic method. Int. J. Mass. Spectr., 2007, 267(1-3), 199-204.

[48] Seco, J.M.; Riguera, R., NMR Methods for the Assignment of Absolute Stereochemistry of Bioactive Compounds. eMagRes, 2015, 4(1), 1-30.

[49] Albright, A.L.; White, J.M. In Metabolomics Tools for Natural Product Discovery: Methods and Protocols. Roessner, U.; Dias, A.D., Eds.; Humana Press: Totowa, NJ, 2013, pp 149-162.

[50] Groom, C.R.; Bruno, I.J.; Lightfoot, M.P.; Ward, S.C., The Cambridge Structural Database. Acta Crystallograph. Sect. B, 2016, 72(2), 171-179.

[51] Flack, H.D.; Bernardinelli, G., The use of X-ray crystallography to determine absolute configuration. Chirality, 2008, 20(5), 681-690.

[52] Pitzer, M.; Kastirke, G.; Kunitski, M.; Jahnke, T.; Bauer, T.; Goihl, C.; Trinter, F.; Schober, C.; Henrichs, K.; Becht, J.; Zeller, S.; Gassert, H.; Waitz, M.; Kuhlins, A.; Sann, H.; Sturm, F.; Wiegandt, F.; Wallauer, R.; Schmidt, L.P.H.; Johnson, A.S.; Mazenauer, M.; Spenger, B.; Marquardt, S.; Marquardt, S.; Schmidt-Böcking, H.; Stohner, J.; Dörner, R.; Schöffler, M.; Berger, R., Absolute Configuration from Different Multifragmentation Pathways in Light-Induced Coulomb Explosion Imaging. ChemPhysChem, 2016, 17(16), 2465-2472.

[53] Patterson, D.; Schnell, M., New studies on molecular chirality in the gas phase: enantiomer differentiation and determination of enantiomeric excess. Phys. Chem. Chem. Phys., 2014, 16(23), 11114-11123.

[54] Berova, N.; Di Bari, L.; Pescitelli, G., Application of electronic circular dichroism in configurational and conformational analysis of organic compounds. Chem. Soc. Rev., 2007, 36(6), 914-931.

[55] Barron, L.D. Molecular Light Scattering and Optical Acticity. Cambridge Univeristy press: Cambridge, 2004.

[56] Sutherland, J.C. In Comprehensive Chiroptical Spectroscopy. Berova, N.; Polavarapu, P.L.; Nakanishi, K.; Woody, R.W., Eds.; John Wiley \& Sons, Inc.: Hoboken, NJ, 2012; Vol. 1, pp 35-63.
[57] Molinski, T.F., Microscale methodology for structure elucidation of natural products. Curr. Opin. Biotechn., 2010, 21(6), 819-826.

[58] Nafie, L. Vibrational Optical Activity, Principles and Applications. John Wiley \& Sons: New York, 2011.

[59] Keiderling, T.A., Protein and peptide secondary structure and conformational determination with vibrational circular dichroism. Curr. Opin. Chem. Biol., 2002, 6(5), 682688.

[60] Nafie, L.A.; Diem, M.; Vidrine, D.W., Fourier transform infrared vibrational circular dichroism. J. Am. Chem. Soc., 1979, 101(2), 496-498.

[61] Abbate, S.; Longhi, G.; Castiglioni, E. In Comprehensive Chiroptical Spectroscopy. Berova, N.; Polavarapu, P.L.; Nakanishi, K.; Woody, R.W., Eds.; John Wiley \& Sons, Inc.: Hoboken, NJ, 2012; Vol. 1, pp 247-273. [62] Stephens, P.J.; Devlin, F.J.; Schürch, S.; Hulliger, J., Determination of the absolute configuration of chiral molecules via density functional theory calculations of vibrational circular dichroism and optical rotation: The chiral alkane D3-anti-trans-anti-trans-anti-transperhydrotriphenylene. Theor. Chem. Acc., 2008, 119(1), 1928.

[63] Superchi, S.; Rosini, C.; Mazzeo, G.; Giorgio, E. In Comprehensive Chiroptical Spectroscopy. Berova, N.; Polavarapu, P.L.; Nakanishi, K.; Woody, R.W., Eds.; John Wiley \& Sons, Inc.: Hoboken, NJ, 2012; Vol. 2, pp 421-447. [64] Pescitelli, G.; Di Bari, L.; Berova, N., Conformational Aspects in the Studies of Organic Compounds by Electronic Circular Dichroism. Chem. Soc. Rev., 2011, 40, 4603-4625.

[65] Yamada, T.; Ohshima, M.; Yuasa, K.; Kikuchi, T.; Tanaka, R., Assignment of the CD Cotton Effect to the Chiral Center in Pseurotins, and the Stereochemical Revision of Pseurotin A2. Marine Drugs, 2016, 14(4), 74.

[66] Zhang, Z.; He, X.; Liu, C.; Che, Q.; Zhu, T.; Gu, Q.; Li, D., Clindanones A and B and cladosporols F and G, polyketides from the deep-sea derived fungus Cladosporium cladosporioides HDN14-342. RSC Advances, 2016, 6(80), 76498-76504.

[67] Kwit, M.; Skowronek, P.; Gawronski, J.; Frelek, J.; Woznica, M.; Butkiewicz, A. In Comprehensive Chiroptical Spectroscopy. Berova, N.; Polavarapu, P.L.; Nakanishi, K.; Woody, R.W., Eds.; John Wiley \& Sons, Inc.: Hoboken, NJ, 2012; Vol. 2, pp 37-72.

[68] Lightner, D.A.; Gurst, J.E. Organic Conformational Analysis and Stereochemistry from Circular Dichroism. Wiley: New York, 2000.

[69] Kurtán, T.; Antus, S.; Pescitelli, G. In Comprehensive Chiroptical Spectroscopy. Berova, N.; Polavarapu, P.L.; Nakanishi, K.; Woody, R.W., Eds.; John Wiley \& Sons, Inc.: Hoboken, NJ, 2012; Vol. 2, pp 73-114.

[70] Kock, I.; Draeger, S.; Schulz, B.; Elsässer, B.; Kurtán, T.; Kenéz, Á.; Antus, S.; Pescitelli, G.; Salvadori, P.; Speakman, J.-B.; Rheinheimer, J.; Krohn, K., 
Pseudoanguillosporin A and B: Two New Isochromans Isolated from the Endophytic Fungus Pseudoanguillospora sp. Eur. J. Org. Chem., 2009, 2009(9), 1427-1434.

[71] Dražić, T.; Roje, M.; Jurin, M.; Pescitelli, G., Synthesis, Separation and Absolute Configuration Determination by ECD Spectroscopy and TDDFT Calculations of 3-Amino- $\beta$-lactams and Derived Guanidines. Eur. J. Org. Chem., 2016, 2016(24), 4189-4199.

[72] Harada, N.; Nakanishi, K.; Berova, N. In Comprehensive Chiroptical Spectroscopy. Berova, N.; Polavarapu, P.L.; Nakanishi, K.; Woody, R.W., Eds.; John Wiley \& Sons, Inc.: Hoboken, NJ, 2012, pp 115-166.

[73] Berova, N.; Ellestad, G.A.; Harada, N. In Comprehensive Natural Products II. Mander, L.; Liu, H.-W., Eds.; Elsevier: Oxford, 2010; Vol. 9, pp 91-146.

[74] Berova, N.; Pescitelli, G.; Petrovic, A.G.; Proni, G., Probing molecular chirality by CD-sensitive dimeric metalloporphyrin hosts. Chem. Commun., 2009(40), 59585980 .

[75] Schlingmann, G.; Matile, S.; Berova, N.; Nakanishi, K.; Carter, G.T., Absolute stereochemistry of the diepoxins. Tetrahedron, 1996, 52(2), 435-446.

[76] Superchi, S.; Giorgio, E.; Rosini, C., Structural determinations by circular dichroism spectra analysis using coupled oscillator methods: An update of the applications of the DeVoe polarizability model. Chirality, 2004, 16(7), 422451 .

[77] Raabe, G.; Fleischhauer, J.; Woody, R.W. In Comprehensive Chiroptical Spectroscopy. Berova, N.; Polavarapu, P.L.; Nakanishi, K.; Woody, R.W., Eds.; John Wiley \& Sons, Inc.: Hoboken, NJ, 2012; Vol. 1, pp 541-591.

[78] Wolf, C.; Bentley, K.W., Chirality sensing using stereodynamic probes with distinct electronic circular dichroism output. Chem. Soc. Rev., 2013, 42(12), 54085424.

[79] Superchi, S.; Casarini, D.; Laurita, A.; Bavoso, A.; Rosini, C., Induction of a Preferred Twist in a Biphenyl Core by Stereogenic Centers: A Novel Approach to the Absolute Configuration of 1,2- and 1,3-Diols. Angew. Chem. Int. Ed., 2001, 40(2), 451-454.

[80] Superchi, S.; Bisaccia, R.; Casarini, D.; Laurita, A.; Rosini, C., Flexible Biphenyl Chromophore as a Circular Dichroism Probe for Assignment of the Absolute Configuration of Carboxylic Acids. J. Am. Chem. Soc., 2006, 128(21), 6893-6902.

[81] Autschbach, J., Computing chiroptical properties with first-principles theoretical methods: Background and illustrative examples. Chirality, 2009, 21(1E), E116-E152.

[82] Autschbach, J. In Comprehensive Chiroptical Spectroscopy. Berova, N.; Polavarapu, P.L.; Nakanishi, K.; Woody, R.W., Eds.; John Wiley \& Sons, Inc.: Hoboken, NJ, 2012, pp 593-642.

[83] Goerigk, L.; Kruse, H.; Grimme, S. In Comprehensive Chiroptical Spectroscopy. Berova, N.;
Polavarapu, P.L.; Nakanishi, K.; Woody, R.W., Eds.; John Wiley \& Sons, Inc.: Hoboken, NJ, 2012, pp 643-673.

[84] Warnke, I.; Furche, F., Circular dichroism: electronic. WiRES Comput. Mol. Sci., 2012, 2(1), 150-166.

[85] Pescitelli, G.; Bruhn, T., Good Computational Practice in the Assignment of Absolute Configurations by TDDFT Calculations of ECD Spectra. Chirality, 2016, 28(6), 466-474.

[86] Autschbach, J.; Nitsch-Velasquez, L.; Rudolph, M., Time-Dependent Density Functional Response Theory for Electronic Chiroptical Properties of Chiral Molecules. Top. Curr. Chem., 2011, 298, 1-98.

[87] Holzwarth, G.; Hsu, E.C.; Mosher, H.S.; Faulkner, T.R.; Moscowitz, A., Infrared circular dichroism of carbonhydrogen and carbon-deuterium stretching modes. Observations. J. Am. Chem. Soc., 1974, 96(1), 251-252.

[88] Holzwarth, G.; Chabay, I., Optical Activity of Vibrational Transitions: A Coupled Oscillator Model. The Journal of Chemical Physics, 1972, 57(4), 1632-1635.

[89] Taniguchi, T.; Monde, K., Exciton Chirality Method in Vibrational Circular Dichroism. J. Am. Chem. Soc., 2012, 134(8), 3695-3698.

[90] Asai, T.; Taniguchi, T.; Yamamoto, T.; Monde, K.; Oshima, Y., Structures of Spiroindicumides A and B, Unprecedented Carbon Skeletal Spirolactones, and Determination of the Absolute Configuration by Vibrational Circular Dichroism Exciton Approach. Org. Lett., 2013, 15(17), 4320-4323.

[91] Yang, G.; Xu, Y. In Electronic and Magnetic Properties of Chiral Molecules and Supramolecular Architectures. Naaman, R.; Beratan, D.N.; Waldeck, D., Eds.; Springer Berlin Heidelberg: Berlin, Heidelberg, 2011, pp 189-236.

[92] Stephens, P.J., Theory of vibrational circular dichroism. J. Phys. Chem., 1985, 89(5), 748-752.

[93] Devlin, F.J.; Stephens, P.J.; Cheeseman, J.R.; Frisch, M.J., Prediction of Vibrational Circular Dichroism Spectra Using Density Functional Theory: Camphor and Fenchone. J. Am. Chem. Soc., 1996, 118(26), 6327-6328.

[94] Kuhn, W., Das Problem der absoluten Konfiguration optisch aktiver Stoffe. Naturwiss., 1938, 26, 305-310.

[95] Djerassi, C. Optical rotatory dispersion: Applications to organic chemistry. McGraw-Hill: New York, 1960.

[96] Amos, R.D., Electric and Magnetic-Properties of $\mathrm{CO}, \mathrm{HF}, \mathrm{HCl}$ and $\mathrm{CH} 3 \mathrm{~F}$. Chem. Phys. Lett., 1982, 87(1), 2326.

[97] Polavarapu, P.L., Ab initio molecular optical rotations and absolute configurations. Mol. Phys., 1997, 91(3), 551-554.

[98] Cheeseman, J.R.; Frisch, M.J.; Devlin, F.J.; Stephens, P.J., Hartree-Fock and Density Functional Theory ab Initio Calculation of Optical Rotation Using GIAOs: 
Basis Set Dependence. J. Phys. Chem. A, 2000, 104(5), 1039-1046.

[99] Stephens, P.J.; Devlin, F.J.; Cheeseman, J.R.; Frisch, M.J., Calculation of Optical Rotation Using Density Functional Theory. J. Phys. Chem. A, 2001, 105(22), 53565371.

[100] Giorgio, E.; Viglione, R.G.; Zanasi, R.; Rosini, C., $\mathrm{Ab}$ Initio Calculation of Optical Rotatory Dispersion (ORD) Curves: A Simple and Reliable Approach to the Assignment of the Molecular Absolute Configuration. J. Am. Chem. Soc., 2004, 126(40), 12968-12976.

[101] Derewacz, D.K.; McNees, C.R.; Scalmani, G.; Covington, C.L.; Shanmugam, G.; Marnett, L.J.; Polavarapu, P.L.; Bachmann, B.O., Structure and Stereochemical Determination of Hypogeamicins from a Cave-Derived Actinomycete. J. Nat. Prod., 2014, 77(8), 1759-1763.

[102] Junior, F.M.S.; Covington, C.L.; de Amorim, M.B.; Velozo, L.S.M.; Kaplan, M.A.C.; Polavarapu, P.L., Absolute Configuration of a Rare Sesquiterpene: (+)-3-Ishwarone. $J$. Nat. Prod., 2014, 77(8), 1881-1886.

[103] Halgren, T.A., Merck molecular force field. I. Basis, form, scope, parameterization, and performance of MMFF94. J. Comput. Chem., 1996, 17(5-6), 490-519 and following papers.

[104] Mazzanti, A.; Casarini, D., Recent trends in conformational analysis. WiRES Comput. Mol. Sci., 2012, 2(4), 613-641.

[105] Hinchliffe, A. Molecular Modelling for Beginners. $2^{\text {nd }}$ ed. ed. Wiley: Chichester, 2008.

[106] Polavarapu, P.L., Molecular Structure Determination Using Chiroptical Spectroscopy: Where We May Go Wrong? Chirality, 2012, 24(11), 909-920.

[107] Brémond, É.; Savarese, M.; Su, N.Q.; PérezJiménez, Á.J.; Xu, X.; Sancho-García, J.C.; Adamo, C., Benchmarking Density Functionals on Structural Parameters of Small-/Medium-Sized Organic Molecules. J. Chem. Theory Comput., 2016, 12(2), 459-465.

[108] Mennucci, B.; Cammi, R., Eds. Continuum Solvation Models in Chemical Physics: From Theory to Applications Wiley: Chichester, 2007.

[109] Casarini, D.; Lunazzi, L.; Mazzanti, A., Recent Advances in Stereodynamics and Conformational Analysis by Dynamic NMR and Theoretical Calculations. Eur. J. Org. Chem., 2010, 2010(11), 2035-2056.

[110] Adamo, C.; Jacquemin, D., The calculations of excited-state properties with Time-Dependent Density Functional Theory. Chem. Soc. Rev., 2013, 42(3), 845-856.

[111] Dreuw, A.; Head-Gordon, M., Single-Reference ab Initio Methods for the Calculation of Excited States of Large Molecules. Chem. Rev., 2005, 105(11), 4009-4037.

[112] Reinscheid, F.; Reinscheid, U.M., Stereochemical analysis of $(+)$-limonene using theoretical and experimental NMR and chiroptical data. J. Mol. Struct., 2016, 1106, 141153.
[113] Mennucci, B.; Cappelli, C.; Cammi, R.; Tomasi, J., Modeling solvent effects on chiroptical properties. Chirality, 2011, 23(9), 717-729.

[114] Stephens, P.J.; Harada, N., ECD cotton effect approximated by the Gaussian curve and other methods. Chirality, 2010, 22(2), 229-233.

[115] Grimme, S.; Mück-Lichtenfeld, C., Calculation of conformational energies and optical rotation of the most simple chiral alkane. Chirality, 2008, 20(9), 1009-1015.

[116] Covington, C.L.; Polavarapu, P.L., Similarity in Dissymmetry Factor Spectra: A Quantitative Measure of Comparison between Experimental and Predicted Vibrational Circular Dichroism. J. Phys. Chem. A, 2013, 117(16), 3377-3386.

[117] Polavarapu, P.L.; Covington, C.L., Comparison of Experimental and Calculated Chiroptical Spectra for Chiral Molecular Structure Determination. Chirality, 2014, 26(9), 539-552.

[118] Bruhn, T.; Schaumlöffel, A.; Hemberger, Y.; Bringmann, G., SpecDis: Quantifying the Comparison of Calculated and Experimental Electronic Circular Dichroism Spectra. Chirality, 2013, 25(4), 243-249.

[119] Berardozzi, R.; Guido, C.A.; Capozzi, M.A.M.; Cardellicchio, C.; Di Bari, L.; Pescitelli, G., Circular Dichroism and TDDFT Investigation of Chiral Fluorinated Aryl Benzyl Sulfoxides. Eur. J. Org. Chem., 2015(25), 5554-5562.

[120] Bannwarth, C.; Grimme, S., A simplified timedependent density functional theory approach for electronic ultraviolet and circular dichroism spectra of very large molecules. Comput. Theor. Chem., 2014, 1040-1041, 45-53.

[121] Sneskov, K.; Christiansen, O., Excited state coupled cluster methods. WiRES Comput. Mol. Sci., 2012, 2(4), 566584.

[122] Grimme, S., Calculation of frequency dependent optical rotation using density functional response theory. Chem. Phys. Lett., 2001, 339(5-6), 380-388.

[123] Srebro, M.; Govind, N.; de Jong, W.A.; Autschbach, J., Optical Rotation Calculated with TimeDependent Density Functional Theory: The OR45 Benchmark. J. Phys. Chem. A, 2011, 115(40), 10930-10949.

[124] SciFinder, American Chemical Society: 2016.

[125] Polavarapu, P.L., Why is it important to simultaneously use more than one chiroptical spectroscopic method for determining the structures of chiral molecules? Chirality, 2008, 20(5), 664-672.

[126] Hussain, H.; Krohn, K.; Ahmed, I.; Draeger, S.; Schulz, B.; Pietro, S.; Pescitelli, G., Phomopsinones A-D: Four New Pyrenocines from Endophytic Fungus Phomopsis sp. Eur. J. Org. Chem., 2012, 2012(9), 1783-1789.

[127] Sun, P.; Xu, D.X.; Mandi, A.; Kurtan, T.; Li, T.J.; Schulz, B.; Zhang, W., Structure, absolute configuration, and conformational study of 12-membered macrolides from the fungus Dendrodochium sp. associated with the sea cucumber 
Holothuria nobilis Selenka. J. Org. Chem., 2013, 78(14), 7030-7047.

[128] Kuroda, R.; Harada, T. In Comprehensive Chiroptical Spectroscopy. Berova, N.; Polavarapu, P.L.; Nakanishi, K.; Woody, R.W., Eds.; John Wiley \& Sons, Inc., 2012; Vol. 1, pp 91-113.

[129] Dai, J.; Hussain, H.; Drager, S.; Schulz, B.; Kurtan, T.; Pescitelli, G.; Florke, U.; Krohn, K., Metabolites from the fungus Phoma sp. 7210, associated with Aizoon canariense. Nat. Prod. Commun., 2010, 5(8), 1175-1180.

[130] Dai, J.Q.; Krohn, K.; Florke, U.; Pescitelli, G.; Kerti, G.; Papp, T.; Kover, K.E.; Benyei, A.C.; Draeger, S.; Schulz, B.; Kurtan, T., Curvularin-Type Metabolites from the Fungus Curvularia sp Isolated from a Marine Alga. Eur. J. Org. Chem., 2010(36), 6928-6937.

[131] Hussain, H.; Ahmed, I.; Schulz, B.; Draeger, S.; Florke, U.; Pescitelli, G.; Krohn, K., Solid-state circular dichroism and hydrogen bonding: absolute configuration of massarigenin A from Microsphaeropsis sp. Chirality, 2011, 23(8), 617-623.

[132] Saleem, M.; Hussain, H.; Ahmed, I.; Draeger, S.; Schulz, B.; Meier, K.; Steinert, M.; Pescitelli, G.; Kurtan, T.; Florke, U.; Krohn, K., Viburspiran, an Antifungal Member of the Octadride Class of Maleic Anhydride Natural Products. Eur. J. Org. Chem., 2011(4), 808-812.

[133] Siddiqui, I.N.; Zahoor, A.; Hussain, H.; Ahmed, I.; Ahmad, V.U.; Padula, D.; Draeger, S.; Schulz, B.; Meier, K.; Steinert, M.; Kurtan, T.; Florke, U.; Pescitelli, G.; Krohn, K., Diversonol and blennolide derivatives from the endophytic fungus Microdiplodia sp.: absolute configuration of diversonol. J. Nat. Prod., 2011, 74(3), 365-373.

[134] Cimmino, A.; Mathieu, V.; Masi, M.; Baroncelli, R.; Boari, A.; Pescitelli, G.; Ferderin, M.; Lisy, R.; Evidente, M.; Tuzi, A.; Zonno, M.C.; Kornienko, A.; Kiss, R.; Evidente, A., Higginsianins A and B, Two Diterpenoid alpha-Pyrones Produced by Colletotrichum higginsianum, with in Vitro Cytostatic Activity. J. Nat. Prod., 2016, 79(1), 116-125.

[135] Elnaggar, M.S.; Ebada, S.S.; Ashour, M.L.; Ebrahim, W.; Mueller, W.E.G.; Mandi, A.; Kurtan, T.; Singab, A.; Lin, W.; Liu, Z.; Proksch, P., Xanthones and sesquiterpene derivatives from a marine-derived fungus Scopulariopsis sp. Tetrahedron, 2016, 72(19), 2411-2419.

[136] Moussa, M.; Ebrahim, W.; El-Neketi, M.; Mandi, A.; Kurtan, T.; Hartmann, R.; Lin, W.; Liu, Z.; Proksch, P., Tetrahydroanthraquinone derivatives from the mangrovederived endophytic fungus Stemphylium globuliferum. Tetrahedron Lett., 2016, 57(36), 4074-4078.

[137] Perez Hemphill, C.F.; Daletos, G.; Hamacher, A.; Kassack, M.U.; Lin, W.; Mandi, A.; Kurtan, T.; Proksch, P., Absolute configuration and antitumor activity of torrubiellin B. Tetrahedron Lett., 2015, 56(30), 4430-4433.

[138] Zhang, P.; Meng, L.-H.; Mandi, A.; Li, X.-M.; Kurtan, T.; Wang, B.-G., Structure, absolute configuration, and conformational study of resorcylic acid derivatives and related congeners from the fungus Penicillium brocae. $R S C$ Adv., 2015, 5(50), 39870-39877.

[139] Lu, S.; Kurtan, T.; Yang, G.J.; Sun, P.; Mandi, A.; Krohn, K.; Draeger, S.; Schulz, B.; Yi, Y.H.; Li, L.; Zhang, W., Cytospolides A-E, New Nonanolides from an Endophytic Fungus, Cytospora sp. Eur. J. Org. Chem., 2011(28), 5452-5459.

[140] Lu, S.; Sun, P.; Li, T.; Kurtan, T.; Mandi, A.; Antus, S.; Krohn, K.; Draeger, S.; Schulz, B.; Yi, Y.; Li, L.; Zhang, W., Bioactive nonanolide derivatives isolated from the endophytic fungus Cytospora sp. J. Org. Chem., 2011, 76(23), 9699-9710.

[141] Giorgio, E.; Maddau, L.; Spanu, E.; Evidente, A.; Rosini, C., Assignment of the absolute configuration of (+)diplopyrone, the main phytotoxin produced by Diplodia mutila, the pathogen of the cork oak decline, by a nonempirical analysis of its chiroptical properties. J. Org. Chem., 2005, 70(1), 7-13.

[142] Evidente, A.; Maddau, L.; Spanu, E.; Franceschini, A.; Lazzaroni, S.; Motta, A., Diplopyrone, a New Phytotoxic Tetrahydropyranpyran-2-one Produced by Diplodia mutila, a Fungus Pathogen of Cork Oak. J. Nat. Prod., 2003, 66(2), 313-315.

[143] Molitor, D.; Liermann, J.C.; Berkelmann-Löhnertz, B.; Buckel, I.; Opatz, T.; Thines, E., Phenguignardic Acid and Guignardic Acid, Phytotoxic Secondary Metabolites from Guignardia bidwellii. J. Nat. Prod., 2012, 75(7), 12651269.

[144] Buckel, I.; Molitor, D.; Liermann, J.C.; Sandjo, L.P.; Berkelmann-Löhnertz, B.; Opatz, T.; Thines, E., Phytotoxic dioxolanone-type secondary metabolites from Guignardia bidwellii. Phytochem., 2013, 89, 96-103.

[145] Andernach, L.; Sandjo, L.P.; Liermann, J.C.; Buckel, I.; Thines, E.; Opatz, T., Assignment of Configuration in a Series of Dioxolanone-Type Secondary Metabolites from Guignardia bidwellii - A Comparison of VCD and ECD Spectroscopy. Eur. J. Org. Chem., 2013, 2013(26), 5946-5951.

[146] Polavarapu, P.L.; Donahue, E.A.; Hammer, K.C.; Raghavan, V.; Shanmugam, G.; Ibnusaud, I.; Nair, D.S.; Gopinath, C.; Habel, D., Chiroptical Spectroscopy of Natural Products: Avoiding the Aggregation Effects of Chiral Carboxylic Acids. J. Nat. Prod., 2012, 75(8), 1441-1450.

[147] Sun, Y.-L.; Bao, J.; Liu, K.-S.; Zhang, X.-Y.; He, F.; Wang, Y.-F.; Nong, X.-H.; Qi, S.-H., Cytotoxic Dihydrothiophene-Condensed Chromones from the MarineDerived Fungus Penicillium oxalicum. Planta Med., 2013, 79(15), 1474-1479.

[148] Wink, C.; Andernach, L.; Opatz, T.; Waldvogel, S.R., Total Synthesis of $( \pm)$-Oxalicumone $C$ and Chiral Resolution and Elucidation of Its Absolute Configuration. Eur. J. Org. Chem., 2014, 2014(35), 7788-7792.

[149] Stephens, P.J.; McCann, D.M.; Devlin, F.J.; Cheeseman, J.R.; Frisch, M.J., Determination of the Absolute Configuration of 
[32](1,4)Barrelenophanedicarbonitrile Using Concerted Time-Dependent Density Functional Theory Calculations of Optical Rotation and Electronic Circular Dichroism. J. Am. Chem. Soc., 2004, 126(24), 7514-7521.

[150] Polavarapu, P.L.; Donahue, E.A.; Shanmugam, G.; Scalmani, G.; Hawkins, E.K.; Rizzo, C.; Ibnusaud, I.; Thomas, G.; Habel, D.; Sebastian, D., A Single Chiroptical Spectroscopic Method May Not Be Able To Establish the Absolute Configurations of Diastereomers: Dimethylesters of Hibiscus and Garcinia Acids. J. Phys. Chem. A, 2011, 115(22), 5665-5673.

[151] Mazzeo, G.; Santoro, E.; Andolfi, A.; Cimmino, A.; Troselj, P.; Petrovic, A.G.; Superchi, S.; Evidente, A.; Berova, N., Absolute configurations of fungal and plant metabolites by chiroptical methods. ORD, ECD, and VCD studies on phyllostin, scytolide, and oxysporone. J. Nat. Prod., 2013, 76(4), 588-599.

[152] Evidente, A.; Cimmino, A.; Andolfi, A.; Vurro, M.; Zonno, M.C.; Motta, A., Phyllostoxin and Phyllostin, Bioactive Metabolites Produced by Phyllosticta cirsii, a Potential Mycoherbicide for Cirsium arvense Biocontrol. $J$. Agric. Food. Chem., 2008, 56(3), 884-888.

[153] Ayer, W.A.; Fukazawa, Y.; Orszanska, H., Scytolide, A New Shikimate Metabolite from the Fungus Scytalidium Uredinicola. Nat. Prod. Lett., 1993, 2(1), 77-82.
[154] Kayode Adesogan, E.; Alo, B.I., Oxysporone, a new metabolite from Fusarium oxysporum. Phytochem., 1979, 18(11), 1886-1887.

[155] Tuzi, A.; Andolfi, A.; Cimmino, A.; Evidente, A., X-Ray Crystal Structure of Phyllostin, a Metabolite Produced by Phyllosticta cirsii, a Potential Mycoherbicide of Cirsium arvense. J. Chem. Crystallogr., 2009, 40(1), 15.

[156] Nicu, V.P.; Baerends, E.J., Robust normal modes in vibrational circular dichroism spectra. Phys. Chem. Chem. Phys., 2009, 11(29), 6107-6118.

[157] Gobi, S.; Magyarfalvi, G., Reliability of computed signs and intensities for vibrational circular dichroism spectra. Phys. Chem. Chem. Phys., 2011, 13(36), 1613016133.

[158] Santoro, E.; Mazzeo, G.; Petrovic, A.G.; Cimmino, A.; Koshoubu, J.; Evidente, A.; Berova, N.; Superchi, S., Absolute configurations of phytotoxins seiricardine A and inuloxin A obtained by chiroptical studies. Phytochem., 2015, 116, 359-366.

[159] Ballio, A.; Castiglione Morelli, M.A.; Evidente, A.; Graniti, A.; Randazzo, G.; Sparapano, L., Seiricardine A, A phytotoxic sesquiterpene from three Seiridium species pathogenic for cypress. Phytochem., 1991, 30(1), 131-136. 